\title{
A metabolic interplay coordinated by HLX regulates myeloid differentiation and $A M L$ through partly overlapping pathways
}

Indre Piragyte1,2, Thomas Clapes (1) 1, Aikaterini Polyzou ${ }^{1,2}$, Ramon I. Klein Geltink ${ }^{3}$, Stylianos Lefkopoulos (iD) 1,2,

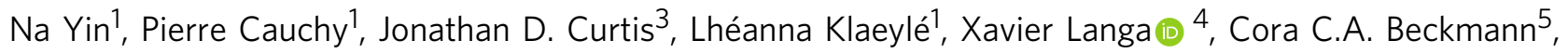
Marcin W. Wlodarski (1] ${ }^{5}$, Patrick Müller [0 ${ }^{6}$, Dominic Van Essen ${ }^{7}$, Angelika Rambold ${ }^{8,9}$, Friedrich G. Kapp ${ }^{5}$, Marina Mione (iD ${ }^{10}$, Joerg M. Buescher (D) ${ }^{3}$, Erika L. Pearce ${ }^{3}$, Alexander Polyzos (D) ${ }^{11}$ \& Eirini Trompouki (i) ${ }^{1}$

The H2.0-like homeobox transcription factor (HLX) regulates hematopoietic differentiation and is overexpressed in Acute Myeloid Leukemia (AML), but the mechanisms underlying these functions remain unclear. We demonstrate here that HLX overexpression leads to a myeloid differentiation block both in zebrafish and human hematopoietic stem and progenitor cells (HSPCs). We show that HLX overexpression leads to downregulation of genes encoding electron transport chain (ETC) components and upregulation of PPARS gene expression in zebrafish and human HSPCs. HLX overexpression also results in AMPK activation. Pharmacological modulation of PPARS signaling relieves the HLX-induced myeloid differentiation block and rescues HSPC loss upon HLX knockdown but it has no effect on AML cell lines. In contrast, AMPK inhibition results in reduced viability of AML cell lines, but minimally affects myeloid progenitors. This newly described role of HLX in regulating the metabolic state of hematopoietic cells may have important therapeutic implications.

\footnotetext{
${ }^{1}$ Department of Cellular and Molecular Immunology, Max Planck Institute of Immunobiology and Epigenetics, 51 Stübeweg, 79108 Freiburg, Germany. ${ }^{2}$ Faculty of Biology, University of Freiburg, Schänzlestraße 1, 79104 Freiburg, Germany. ${ }^{3}$ Department of Immunometabolism, Max Planck Institute of Immunobiology and Epigenetics, 51 Stübeweg, 79108 Freiburg, Germany. ${ }^{4}$ Institute of Anatomy, University of Bern, Baltzerstrasse 2, 3012 Bern, Switzerland. ${ }^{5}$ Division of Pediatric Hematology and Oncology, Department of Pediatrics and Adolescent Medicine, Medical Center - University of Freiburg, Faculty of Medicine, University of Freiburg, Mathildenstr. 1, 79106 Freiburg, Germany. ${ }^{6}$ Systems Biology of Development Group, Friedrich Miescher Laboratory of the Max Planck Society, Max-Planck-Ring 9, 72076 Tübingen, Germany. ${ }^{7}$ Institute for Research on Cancer and Aging Nice, 28 Ave de Valombrose, 06107 Nice Cedex 02, France. ${ }^{8}$ Department of Developmental Immunology, Max Planck Institute of Immunobiology and Epigenetics, 51 Stübeweg, 79108 Freiburg, Germany. ${ }^{9}$ Center for Chronic Immunodeficiency, Freiburg University Medical Center, 55 Hugstetter Street, 79106 Freiburg, Germany. ${ }^{10}$ Centre for Integrative Biology, University of Trento, Via Sommarive, 9, 38123 Povo Trento, Italy. ${ }^{11}$ Biomedical Research Foundation of the Academy of Athens, 4 Soranou Ephessiou Street, 11527 Athens, Greece. These authors contributed equally: Indre Piragyte, Thomas Clapes, Aikaterini Polyzou. Correspondence and requests for materials should be addressed to E.T. (email: trompouki@ie-freiburg.mpg.de)
} 
ong-term hematopoietic stem cells (LT-HSCs) are multipotent cells with self-renewal capacity primarily responsible for replenishing the entire hematopoietic system ${ }^{1-7}$. LTHSC differentiation into mature blood and immune cells is a tightly regulated and multifaceted process. Transcription factors govern the mechanisms that maintain the balance between LTHSC differentiation and self-renewal, or stemness ${ }^{8-10}$, and any perturbation in this process can ultimately lead to disease.

While it is well established that homeobox (HOX) transcription factors play a central role in hematopoietic development and disease, less is known about the function of non-clustered HOX factors in the hematopoietic system ${ }^{11,12}$. The non-clustered H2.0like homeobox transcription factor (HLX) has been recently identified as an important regulator of hematopoiesis. During development, HLX deficiency leads to a decrease in the colonyforming capacity of fetal liver cells ${ }^{13-16}$, and in adult hematopoiesis HLX regulates Th1/Th2 differentiation during T-cell development ${ }^{17-20}$. Recent evidence shows that HLX is essential for HSC maintenance and self-renewal ${ }^{21-23}$. Increased expression of HLX compromises self-renewal and eventually results in a myelomonocytic differentiation block concomitant with aberrant proliferation of myeloid progenitors ${ }^{21}$. Mechanistically, it has been suggested that this function of HLX in HSC maintenance and self-renewal is mediated by the p21-activated kinase PAK1. Indeed, it was demonstrated that inhibition of HLX or PAK1 induces differentiation and apoptosis of AML cells ${ }^{21,22}$. Consistent with this phenotype, HLX is overexpressed in $87 \%$ of AML patients and those presenting higher HLX expression have lower survival rates ${ }^{21}$. Recently, HLX has been shown to play a role in the browning of white adipose tissue, suggesting that this transcription factor is involved in the metabolic control of cell differentiation $^{24}$.

Despite the pleiotropic functions of HLX and its critical regulatory role in multiple processes, particularly in hematopoiesis, only few direct downstream targets have been identified. Moreover, mechanistic insights into the function of HLX in hematopoiesis and myeloid differentiation are lacking. Thus, understanding the physiological roles of HLX in hematopoietic development and disease, including leukemia, remains a central issue in HSC biology.

Here, we use zebrafish, human hematopoietic stem and progenitor cells (HSPCs), and AML cell lines to explore the underlying mechanisms of HLX function during hematopoiesis. We show that HLX overexpression results in an aberrant proliferation of HSPCs and a myeloid differentiation block in both systems. We find that HLX exerts its biological function in hematopoiesis, at least in part, by direct control of electron transport chain (ETC) and PPAR $\delta$ gene expression. Metabolic stress leads to an elevation of AMP-activated kinase (AMPK) levels and autophagy. Modulation of PPAR $\delta$ signaling can rescue the hematopoietic phenotypes of HLX in both zebrafish and human cells, but has no obvious impact on AML cells. In contrast, AMPK inhibition reduces viability of AML cell lines, but minimally affects primary cells. This newly discovered link between HLX and metabolism could be a promising new avenue for treating hematological diseases.

\section{Results}

$H L X$ overexpression blocks zebrafish myeloid cell maturation. To investigate the mechanisms underlying the role of HLX in promoting AML, we examined hematopoiesis in HLXoverexpressing zebrafish models. We crossed the Tg(flila: Gal4FF $) u b s 3^{25}$ line with our $\operatorname{Tg}(U A S: H L X-G F P)$ to induce expression of human $H L X(\mathrm{~h} H L X)$ in endothelial and hematopoietic cells and named these fish fli:hHLXOE. We chose to use human $H L X$ in an effort to demonstrate conservation and translate our results into the human gene function. fli:hHLX overexpression led to increased specification of HSPCs at $36 \mathrm{~h}$ post fertilization (hpf) in the Aorta-Gonad-Mesonephros region as shown by runx 1 whole-mount in situ hybridization (WISH) (Fig. 1a and Supplementary Fig. 1a). The increased number of HSPCs led to increased ragl staining in the thymus at $96 \mathrm{hpf}$ (Fig. 1b). WISH for the early myeloid marker pu.1 revealed that these transgenic fish presented an expansion of myeloid progenitors (Fig. 1c). We then asked whether HLX overexpression affects myeloid cell maturation. May-Grünwald-Giemsa staining showed that fli:hHLXOE embryos have a significantly larger proportion of immature myeloid cells $(75.5 \%)$ when compared to their wild-type counterparts (35.3\%) at $48 \mathrm{hpf}$ (Fig. 1d). EdU staining revealed hyperproliferation of endothelial cells, which may be the underlying cause of the increased number of HSPCs (Fig. 1e). This enhanced proliferation does not induce apoptosis in fli:hHLXOE embryos, as shown by TUNEL assay (Supplementary Fig. 1b).

At $48 \mathrm{hpf}$ most of the myeloid cells are derived from primitive/ prodefinitive and not definitive hematopoiesis. To verify that the differentiation block occurs in myeloid cells that arise from HSPCs we crossed $\operatorname{Tg}(M m u . R u n x 1: G A L 4)$ fish to $T g(U A S: H L X-$ GFP) and named the progeny Runx:hHLXOE. These fish express $\mathrm{hHLX}$ only in HSPCs. In this model we show that more HSPCs are specified at $26 \mathrm{hpf}$ as indicated by runx 1 staining, followed by modestly elevated $c-m y b$ staining and mRNA expression at $3 \mathrm{dpf}$ (days post fertilization) (Supplementary Fig. 1c, d). We then verified by May-Grünwald-Giemsa staining that at $5 \mathrm{dpf}$ HLX overexpression in HSPCs leads to a strong myeloid differentiation block without affecting erythrocyte numbers (Fig. 1f). This result was verified by qPCR and WISH for a panel of mature myeloid markers and gatal as a marker of erythroid differentiation (Supplementary Fig. 1d, e).

Together, these results suggest that HLX overexpression results in increased numbers of HSPCs and blocks myeloid cell differentiation.

HLX is required for HSPC formation. To examine hematopoiesis in $h l x l$ knockdown animals, we generated $h l x l$ morphants ( $h l x 1 \mathrm{MO})$ using a previously published translational morpholino ${ }^{26}$. Inhibition of $h l x 1$ translation in zebrafish embryos decreased the pool of HSPCs, as shown by runxl and $c-m y b$ WISH at $36 \mathrm{hpf}$, respectively (Fig. 1g and Supplementary Fig. 1a, f). To quantify the number of HSPCs in hlx $1 \mathrm{MO}$ animals, we injected either the translational morpholino used in all experiments, or a splicing morpholino ${ }^{26}$ in $\operatorname{Tg}\left(\right.$ Runx:mCherry) $\mathrm{fish}^{27}$, a line with fluorescent HSPCs. The number of m-Cherry ${ }^{+}$cells (HSPCs) was significantly decreased in both types of morphants (Supplementary Fig. 1g). WISH for ragl showed that hlx1MO have fewer thymocytes at $96 \mathrm{hpf}$ (Fig. 1g), when compared to the control embryos. To exclude the possibility that HSPC loss is due to arterial or vascular endothelial defects, we analyzed the expression of arterial (ephrinB2 $\alpha)$ and endothelial $(k d r l)$ markers in $h l x 1 \mathrm{MO}$. In agreement with previous reports, deregulation of $h l x 1$ affects the identity of stack cells, but does not seem to have severe effects on the cardinal vein (Supplementary Fig. 1h) ${ }^{26}$. Additionally, TUNEL assays demonstrated that HSPC loss in $h l \times 1 \mathrm{MO}$ is not caused by apoptosis, whereas $E d U$ staining showed reduced proliferation of endothelial cells (Supplementary Fig. 1b, and Fig. 1e, respectively).

We then attempted to rescue the morphant phenotype by overexpressing $\mathrm{h} H L X$ in endothelial/hematopoietic $(f l i: \mathrm{h} H L X)$ or hemogenic endothelial cells (Runx:hHLX). Remarkably, hHLX 
overexpression in both cell types rescued HSPC loss (Fig. 1h and Supplementary Fig. 1i).

Collectively, these data show that HLX regulates the formation of HSPCs.

HLX regulates genes involved in metabolism. To understand the mechanisms of HLX function in hematopoiesis, we performed RNA-Seq on FACS-sorted endothelial cells from fli:hHLXOE (fli: kaede) and hlx1MO (kdrl:GFP) embryos at $48 \mathrm{hpf}$. Compared with control embryos, we identified 2950 downregulated and
3419 upregulated genes that changed by more than two-fold (negative binomial test (NBT), $P<0.05)$ in $f l i$ hHLXOE embryos (Fig. 2a, Supplementary Fig. 2a, and Supplementary Data 1). On the other hand, 942 genes were downregulated and 1162 genes upregulated over two-fold (NBT, $P<0.05)$ in $h l x 1 \mathrm{MO}$ animals (Fig. 2a, Supplementary Fig. 2a, and Supplementary Data 1). Seventy-nine ( $8 \%$, hypergeometric test (hg.t), $P<0.01)$ of the downregulated genes in $h l x 1 \mathrm{MO}$ were inversely correlated in $f l$ : $\mathrm{hHLXOE}$. In total 869 genes were deregulated in both $h l \times 1 \mathrm{MO}$ and $f l i$ hHLXOE (hg.t, $P<2.27 \mathrm{E}-120$ ). Next, to identify pathways a
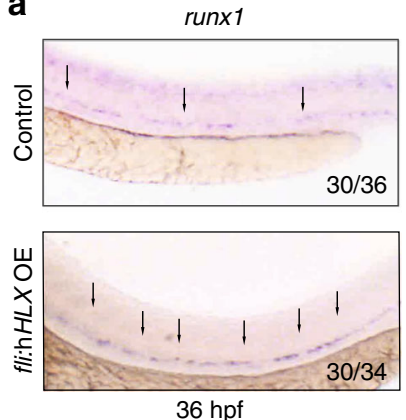

C
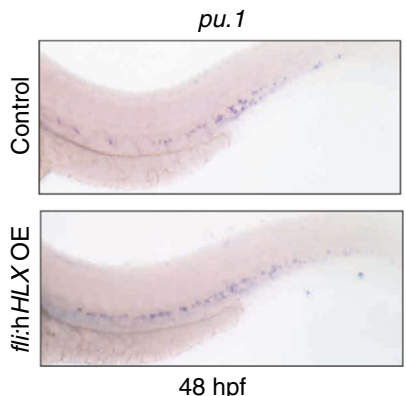

e

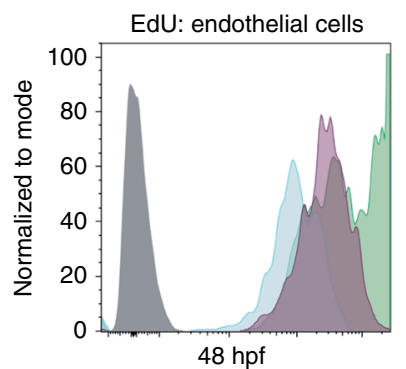

g
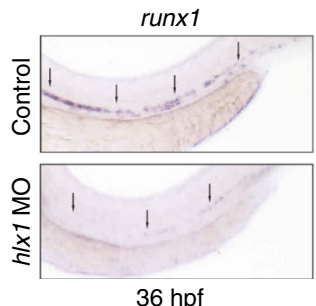

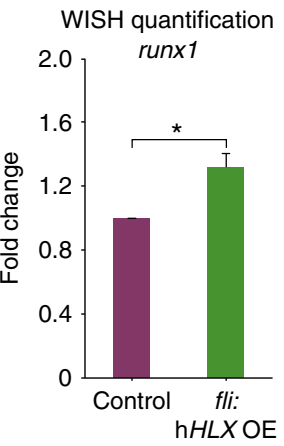

WISH quantification

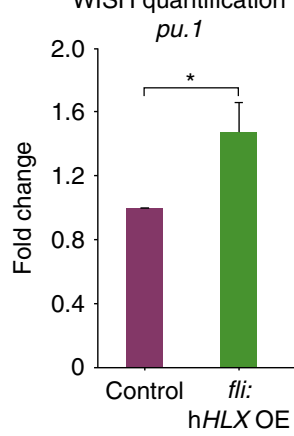

b

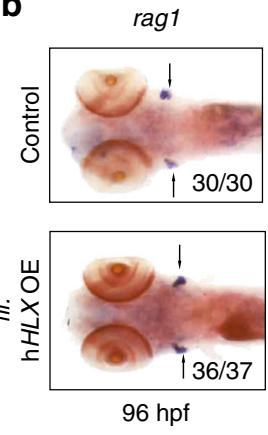

d
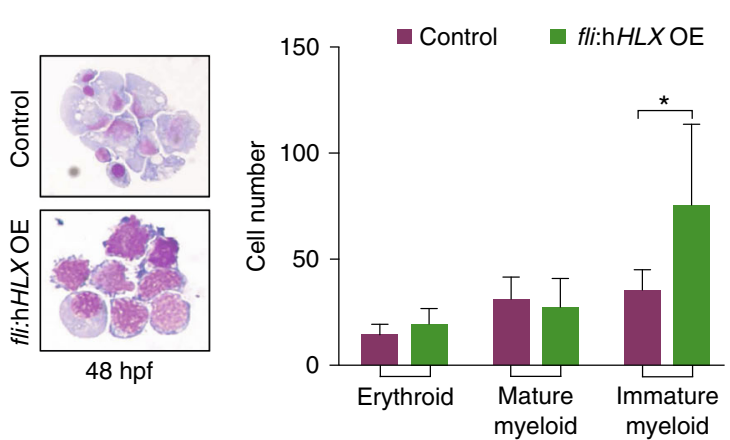

f

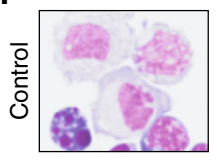

$\square$ Control

$\square / x 1 \mathrm{MO}$

$\square$ fli:hHLXOE
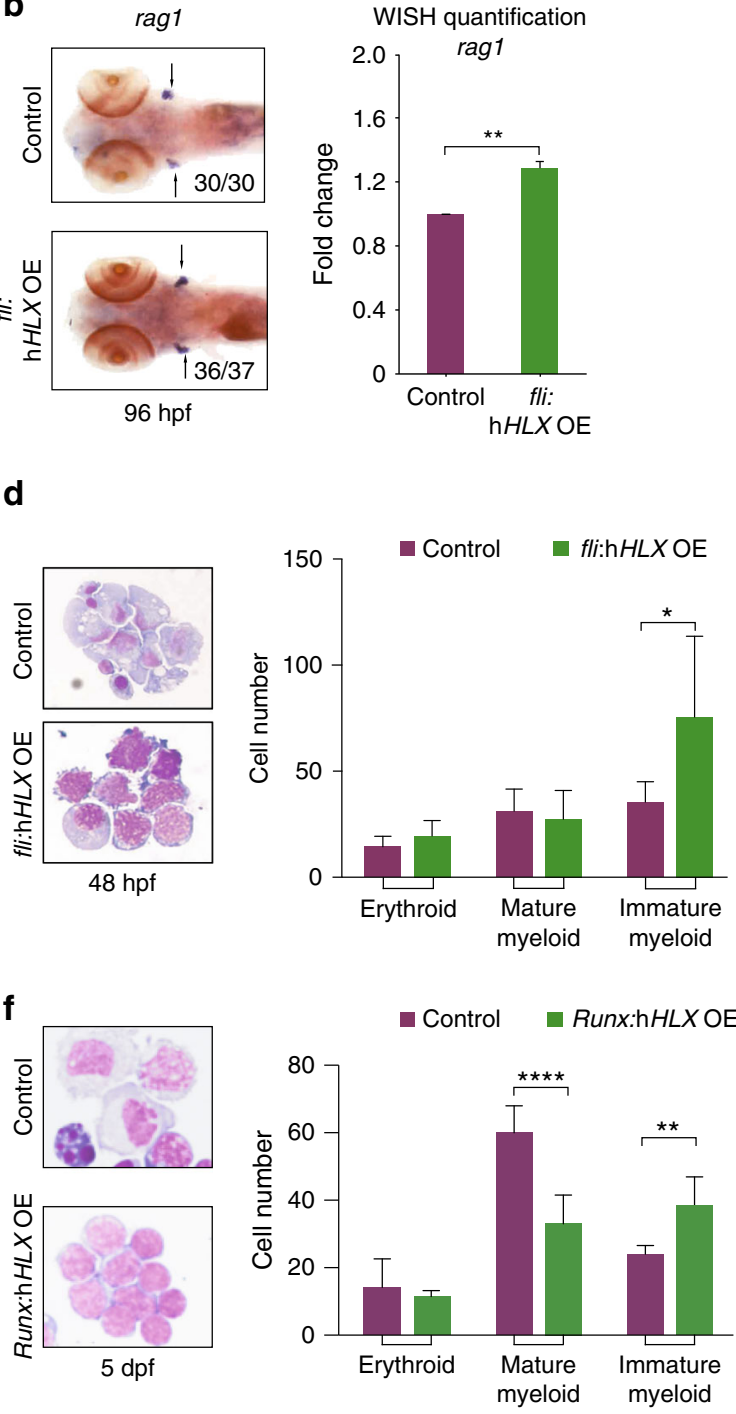

h
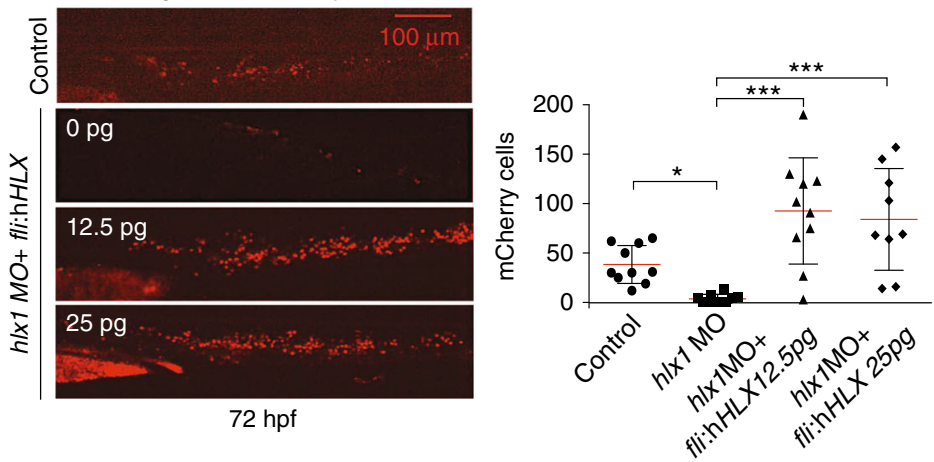
deregulated upon $\mathrm{h} H L X$ overexpression or $h l x 1$ knockdown, we performed ingenuity pathway analysis (IPA), gene ontology, and gene set enrichment analysis (GSEA) and created networks using Cytoscape (Fig. 2b, Supplementary Fig. 2b, c, and Supplementary Data 1). These analyses showed that HLX is a pleiotropic transcription factor that regulates fundamental processes. Interestingly, the two canonical pathways most affected by $\mathrm{h} H L X$ overexpression were oxidative phosphorylation (OXPHOS) (right-tailed Fisher Exact Test (rtFET), $P<3.16 \mathrm{E}-23$ ) and mitochondrial dysfunction (rtFET, $P<1.99 \mathrm{E}-21$, Fig. $2 \mathrm{~b}$ and Supplementary Data 1). GSEA also indicated that genes downregulated in fli:hHLXOE or deregulated in $h l \times 1 \mathrm{MO}$ are associated with changes in mediators of OXPHOS (Supplementary Fig. 2c). Multiple genes of the mitochondrial ETC belonged to the abovementioned categories and were downregulated in fli:hHLXOE embryos (Supplementary Data 1). Gene deregulation detected by RNA-Seq was confirmed by qPCR in fli:hHLXOE and Runx: $\mathrm{h} H L X O E$ or $h l \times 1 \mathrm{MO}$ embryos (Supplementary Fig. 2d-f). These results suggest that HLX regulates mitochondrial metabolic genes. This unexpected finding has important implications, as mitochondrial metabolism is essential for LT-HSCs stemness ${ }^{28,29}$ and AML patients can present defects in mitochondrial metabolism ${ }^{30}$.

Consistent with the transcriptional deregulation observed in the hlx1MO, we also detected differences in chromatin accessibility, by performing ATAC-Seq in endothelial ( $k d r l: G F P)$ cells sorted from control and hlx $1 \mathrm{MO}$ embryos at $48 \mathrm{hpf}$. Using the MACS2 (version 2.1.0) bdgdiff command with the default settings we identified 16,409 peaks that were either lost or gained in hlx $1 \mathrm{MO}$, when compared to wild-type siblings (Fig. $2 \mathrm{c}$ and Supplementary Data 2). Using Genomic Regions Enrichment of Annotations Tool (GREAT) analysis, we found that the majority of peaks were located between 5 and $500 \mathrm{kB}$ of the transcription start site (TSS) and regulate a variety of processes (Supplementary Fig. 3a and Supplementary Data 2). We used a nominal cut-off of $25 \mathrm{~kb}$ from the TSS and assigned the differential peaks to 6431 genes (Supplementary Data 2). IPA analysis of these genes revealed that mitochondrial dysfunction (rtFET, $P<3.47 \mathrm{E}-09$ ) and OXPHOS (rtFET, $P<8.13 \mathrm{E}-08$ ) were among the upper enriched categories (Supplementary Data 2). To gain mechanistic insights we performed footprinting analysis ${ }^{31}$ in control and hlxiMO datasets and obtained 54,588 and 50,471 footprints, respectively. Motif discovery revealed HOX motifs enriched in control-only footprints and AP-1 motifs in hlx1MO-only fooptrints (Supplementary Fig. 3b). To determine whether these motifs were significantly differentially footprinted between the two datasets, we computed motif self-enrichments and cooccurrence enrichments from specific footprint populations over background occurrences computed from reciprocal datasets. This analysis revealed loss of HOX motifs and gain of AP-1 motifs in hlx $1 \mathrm{MO}$ (co-occurrence enrichment computation $z=3.583$ and $z=13.241$, respectively) (Supplementary Fig. 3c). Tn5 insertion profiles at these sites revealed diverging profiles at footprinted Hoxc9 and AP1 motifs (Student's $t$-test, Hoxc9 control specific $P=2.9884 \mathrm{E}-34, \quad$ AP- $1 \quad h l x 1 \mathrm{MO}$ specific $P=1.24262 \mathrm{E}-37$ ) (Fig. 2d). Analysis of relative footprint occurrences to their cognate datasets also revealed that Hoxc9 and AP-1 motifs were more present in control and $h l \times 1 \mathrm{MO}-$ specific footprints, respectively (Supplementary Fig. 3d).

After integration of our RNA-Seq and ATAC-Seq data from the hlx1MO, we identified 739 (35\%, hg.t., $P<1.930 \mathrm{E}-62)$ deregulated genes with changes in chromatin accessibility (Fig. 2e and Supplementary Data 2). Comparison of the ATAC-Seq from $h l \times 1 M O$ and RNA-Seq from fli:hHLXOE embryos demonstrated that 1963 (31\%, hg.t., $P<3.082 \mathrm{e}-116)$ deregulated genes exhibited differences in chromatin accessibility (Fig. 2e, Supplementary Fig. 3e, and Supplementary Data 2). Interestingly, ETC genes that were downregulated upon $\mathrm{h} H L X$ overexpression also showed changes in chromatin accessibility in two different types of ATAC-Seq analyses (whole region or sub-nucleosomal) (Fig. $2 \mathrm{f}$ and Supplementary Data 2). Concomitantly, some ETC genes showed upregulation of expression in hlx1MO (Fig. 2f).

Finally, ppardb (peroxisome-proliferator activated receptor $\delta$ ) also showed changes in chromatin accessibility in subnucleosomal analysis (Fig. 2e and Supplementary Data 2). Recently PPAR $\delta$, a regulator of metabolic functions, was shown to be critical for LT-HSC stemness ${ }^{32}$. We therefore performed qPCR for ppar genes in hxllMO, fli:hHLXOE, and Runx: $\mathrm{hHLXOE}$ embryos. Indeed, the expression of the ppar receptors was significantly decreased in hxllMO (Fig. $2 \mathrm{~g}$ ), but only pparda and ppardb were increased in fli:hHLXOE or Runx:hHLXOE embryos (Fig. 2h, i).

Together, these data demonstrate that Hlx1 regulates the expression of ETC and ppar genes in zebrafish endothelial cells and HSPCs.

PPAR $\delta$ signaling rescues zebrafish hematopoietic phenotypes. The results above suggest that HLX regulates genes involved in metabolism. To investigate this further, we asked whether HLX modulation has any functional consequences for cell metabolism in vivo. We assessed OXPHOS by seahorse metabolic flux analysis, which measures oxygen consumption rate (OCR). These experiments revealed that there is a reduction in spare respiratory capacity in fli:hHLXOE embryos (Fig. 3a). Given the transcriptional deregulation of ETC genes upon hHLX overexpression, we measured mitochondrial membrane potential by using tetramethylrhodamine ethyl ester perchlorate (TMRM). TMRM is a dye that is sequestered in active mitochondria, and reflects the ability of cells to produce ATP. As expected, although the total mitochondrial mass was unchanged in fli:hHLXOE embryos, TMRM was significantly lower (Fig. 3b). In contrast, TMRM was higher in endothelial cells of $h l \times 1 \mathrm{MO}$, yet the total mitochondrial

Fig. $1 \mathrm{hlx} 1$ regulates hematopoietic stem cell formation and myeloid cell maturation in zebrafish. a-b Whole-mount in situ hybridization (WISH) for runx 1 (a) and ragl (b) in control or fli:hHLXOE zebrafish embryos at 36 or $96 \mathrm{hpf}$, respectively. Arrows indicate HSPCs. Numbers in the bottom right corner of panels indicate the number of zebrafish embryos with the indicated phenotype compared to the total number of zebrafish analyzed. Quantification of WISH was performed using FIJI software and statistical significance of three independent experiments in 12 zebrafish embryos was evaluated by Student's $t$-test, ${ }^{\star} P<0.05,{ }^{\star \star} P<0.01$ (mean + s.d.). c WISH for the early myeloid marker pu.1 in control or fli:hHLXOE zebrafish embryos at 48 hpf. Numbers and WISH quantification was performed as described above $\left(n=3\right.$, in total 12 fish, Student's $t$-test, ${ }^{\star} P<0.05$, mean + s.d.). d Zebrafish caudal hematopoietic tissue $(\mathrm{CHT})$ smears stained with May-Grünwald-Giemsa stain in control or fli:hHLXOE embryos at $48 \mathrm{hpf}$. On the right, cell number counts of the indicated cell populations from 10 fish $\left(n=3\right.$, mean + s.d., Student's $t$-test, $\left.{ }^{\star} P<0.05\right)$. e EDU assay at 48 hpf in control, $h / x 1$ morphants ( $\left.h / \times 1 M O\right)$ or fli:hHLXOE zebrafish cells $(n=2)$. f Zebrafish CHT smears stained with May-Grünwald-Giemsa stain in control or Runx:hHLXOE embryos at $5 \mathrm{dpf}$. On the right, cell number counts of the indicated cell populations from 10 fishes, in two independent experiments (mean + s.d., Student's $t$-test, ${ }^{\star \star} P<0.01,{ }^{\star \star \star \star} P<0.0001$ ). $\mathbf{g}$ WISH for runx1 and rag1 in control or hlx1MO embryos at 36 and 96 hpf, respectively. $\mathbf{h}$ Representative images of $T g$ (Runx:mCherry) embryos at $72 \mathrm{hpf}$, injected or not with $h / x 1$ morpholino and the indicated amounts of fli:hHLX construct. Numbers of mCherry-positive HSPCs from each embryo are represented in the graph $\left(n=10\right.$; mean + s.d., Student's $t$-test, $\left.{ }^{\star} P<0.05,{ }^{\star \star \star} P<0.001\right)$ 
d

a

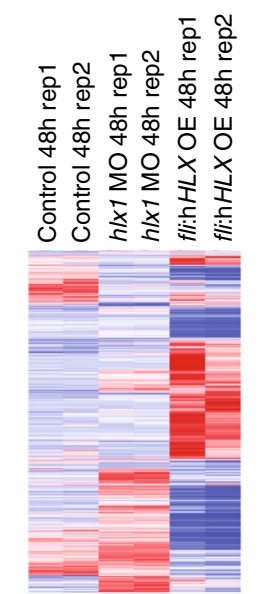

$Z$-transformed normalized gene expression values

$\begin{array}{lll}-1.5 & 0 & 1.5\end{array}$ b

\begin{tabular}{lc} 
Canonical pathways: $48 \mathrm{hpf}$ fli:hHLX OE \\
\hline Name & $P$-value \\
\hline Oxidative phosphorylation & $3.16 \mathrm{E}-23$ \\
Mitochondrial dysfuncion & $1.99 \mathrm{E}-21$ \\
Protein ubiquitination pathway & $1.00 \mathrm{E}-12$ \\
Estrogen receptor signaling & $1.10 \mathrm{E}-08$ \\
Axonal guidance signaling & $1.02 \mathrm{E}-07$
\end{tabular}

\section{Canonical pathways: 48 hpf $h / x 1$ MO}

Name

tRNA charging

FXR/RXR activation

Unfolded protein response
EIF2 signaling

P-value

$P$-value

$1.07 \mathrm{E}-08$

$2.40 \mathrm{E}-03$

$5.50 \mathrm{E}-03$

$1.46 \mathrm{E}-02$

3.72E-02

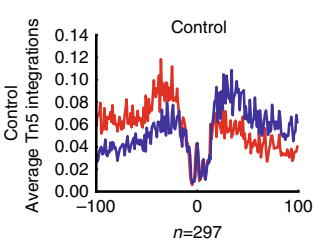

Hoxc9 footprints
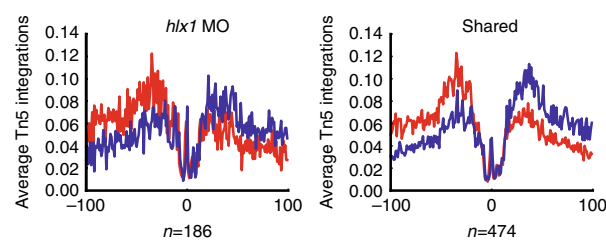

AP-1 footprints
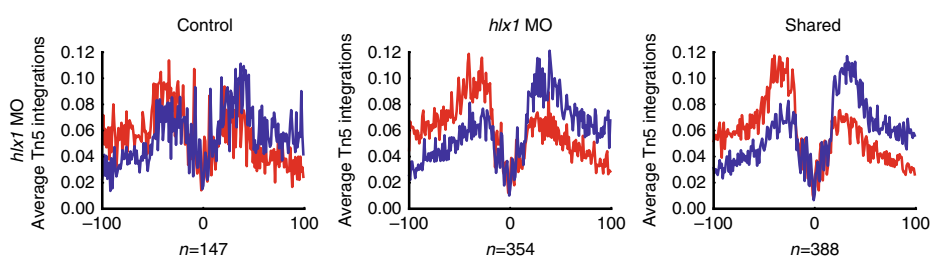

c
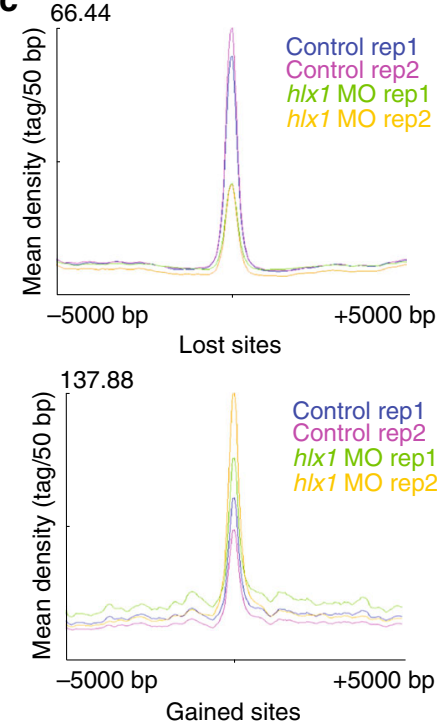

e
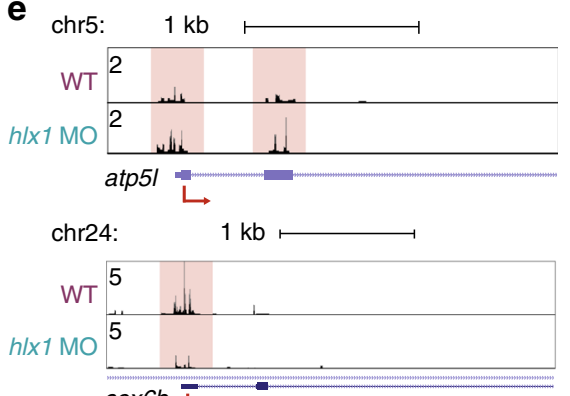

chr3:
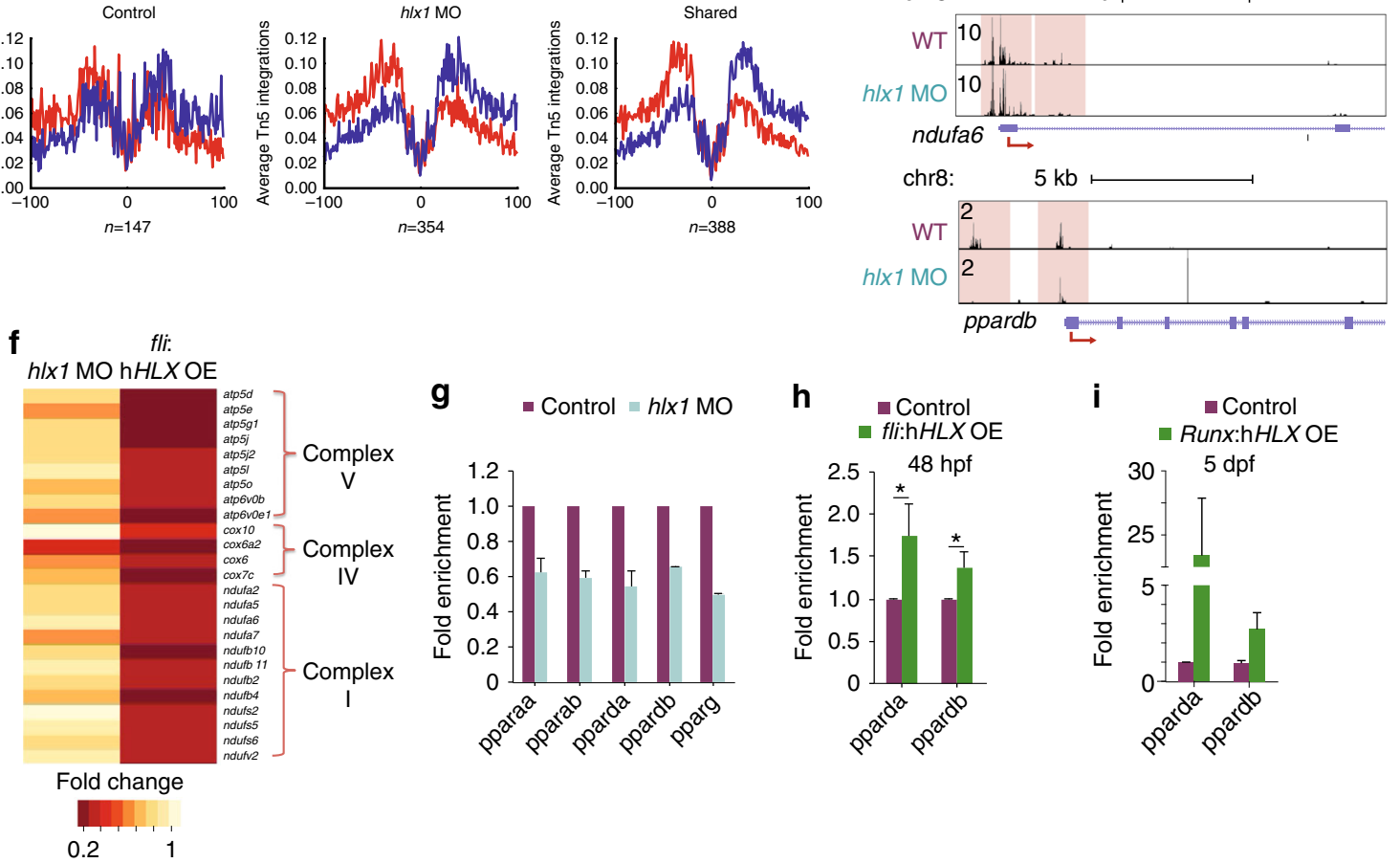

\section{h}

- Control - fli:hHLXOE

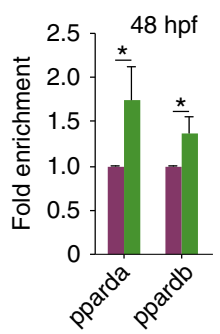

i

- Control - RUnx:hHLXOE

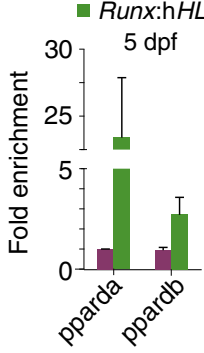

mass was slightly decreased (Fig. 3c). These data demonstrate that HLX overexpression affects mediators of OXPHOS and mitochondrial membrane potential in zebrafish in vivo.

As HLX regulates PPAR $\delta$ gene transcription, a gene essential for lipid metabolism ${ }^{33}$, and in some systems mitochondrial biogenesis ${ }^{34}$, we assessed whether pharmacological modulation of PPAR $\delta$ can rescue defects in mitochondrial membrane potential maintenance. Indeed, we were able to rescue TMRM levels by using a specific PPAR $\delta$ antagonist (GSK3787) in fli:hHLXOE embryos, accompanied by an increase in the mitochondrial mass 
(Fig. 3d). This PPAR $\delta$ antagonist also elevated the TMRM of the control cells, albeit to a lower extent. We then attempted to rescue the HLX-induced hematopoietic phenotypes by modulating the activity of PPAR $\delta$. First, we treated fli:hHLXOE zebrafish embryos with a PPAR $\delta$ antagonist (GSK3787) and examined erythro-myeloid cells at $48 \mathrm{hpf}$. Pharmacological inhibition of PPAR $\delta$ significantly reduced the number of immature myeloid cells and partially rescued the myeloid block (Fig. 3e). Conversely, a PPAR $\delta$ agonist $(\mathrm{L} 165,041)$ rescued HSPC loss in $h l \times 1 \mathrm{MO}$ embryos (Fig. 3f).

These results demonstrate that modulation of PPAR $\delta$ rescues HLX-induced defects in HSPC formation and myeloid cell maturation.

HLX regulates ETC and PPAR genes in human cells. We next addressed the physiological relevance of the interplay between HLX and metabolic regulation in humans. From the database BloodSpot $^{35}$ and previously published data ${ }^{36,37}$, we confirmed that HLX is expressed in murine and human hematopoietic progenitor cells, but also in mature myeloid lineages (Supplementary Fig. $4 \mathrm{a}-\mathrm{c}$ ). Moreover, HLX is upregulated in AML patient samples, as it has been previously published ${ }^{21}$ and according to information from cBioportal (Supplementary Fig. 4d). We next investigated whether AML patients show similar transcriptional signatures to those of hHLXOE zebrafish embryos. We obtained data generated by the TCGA Research Network (http://cancergenome.nih.gov/) from 193 AML patients. Normalized gene expression data of 156 AML samples were used for further analysis. We performed correlation analysis among all genes with significant expression (RSEM values $>50$ ) across all patients and the HLX gene. We then did GO and IPA analyses (Supplementary Data 3 ) in the $H L X$ positively (Pearson Correlation Coefficient $>0.4,824$ genes) and negatively (Pearson Correlation Coefficient $<-0.4,542$ genes) correlated genes. Consistent with the zebrafish data, OXPHOS (rtFET, $P<0.0003$ ), mitochondrial dysfunction (rtFET, $P<0.0107$ ), and other metabolic categories were among the pathways that showed positive correlation with HLX expression in AML patients in IPA analysis (Supplementary Fig. 4e and Data 3). Interestingly, HLX expression in patients positively correlates with PPAR $\delta$ expression (Pearson Correlation Coefficient 0.42) (Supplementary Data 3).

These genomic analyses in AML patients suggest that the role of HLX in metabolic regulation is conserved in humans. To identify genes that are directly regulated by HLX in human hematopoietic cells, we performed ChIP-Seq in two mammalian cell lines (chronic myelogenous leukemia CML: K562 and acute myeloid leukemia-AML HL60) overexpressing a FLAG-tagged version of hHLX (Supplementary Fig. 5a). In both cell lines, the majority of HLX ChIP-Seq peaks fell in introns (48.7\% in K562 and $50.2 \%$ in HL60) and 50-500 kb from the TSS (Supplementary Fig. 5b). After assigning the peaks located within $5 \mathrm{~kb}$ of the TSS to their corresponding genes, we found that HLX was bound to
2135 and 6838 genes in K562 and HL60 cells, respectively. 1689 genes were found in both cell lines (hg.t., $P<0$ ) (Supplementary Fig. 5c and Supplementary Data 4). Notably, 421 (19\%, hg.t., $P<$ 7.044E-10) and 1431 (21\%, hg.t., $P<1.088 \mathrm{E}-53)$ bound genes in K562 and HL60 cells, respectively, also showed differential ATAC-Seq peaks in zebrafish. GO, IPA, Cytoscape, and GREAT analyses revealed that, similar to zebrafish, HLX directly regulates basic cellular processes, including metabolic pathways (Supplementary Fig. 5d and Supplementary Data 4). IPA analyses in K562 and HL60 cells demonstrated that HLX regulates mitochondrial and PPAR/RXR pathways (Fig. 4a). Indeed, multiple ETC genes, but also $P P A R \delta$, were directly bound by HLX in either or both cell types on regions with characteristics of enhancers as indicated by $\mathrm{H} 3 \mathrm{~K} 4 \mathrm{mel}$ and other histone marks (Fig. 4b and Supplementary Data 4). We confirmed these results by ChIP-qPCR on independent ChIP experiments with FLAGtagged or HA-tagged constructs (Fig. 4c). We also performed ChIP-qPCR for HLX target genes on K562 cells carrying an endogenous 3xTy tag on the HLX gene (Fig. 4d). Deletion of one of the bound regions in the vicinity of the $A T P 11 b$ gene using CRISPR-Cas9 technology confirmed that HLX-bound regions can affect gene expression (Supplementary Fig. 5e). Binding motifs for multiple transcription factors were identified in both K562 and HL60 cells (Fig. 4e and Supplementary Data 4). Importantly, independent motif analysis uncovered motifs for homeobox containing factors such as HMBOX1 (Fig. 4e and Supplementary Data 4). To unravel the chromatin landscape around the HLXbound genomic regions, we analyzed available K562 chromatin data from the ENCODE database ${ }^{38}$. HLX-bound genomic regions were located mostly on open chromatin and active enhancers, as indicated by co-localization with active histone marks (Fig. 4f).

Next, we asked whether HLX binding is associated with changes in gene expression in human hematopoietic cells. To this end, we knocked out HLX in K562 cells using CRISPR-Cas9 technology and performed RNA-Seq analysis (Supplementary Fig. 5f). We found that 1324 genes were downregulated and 600 genes were upregulated in $H L X$ knockout cells ( $>2$-fold change, NBT, $P<0.05$, Fig. $4 \mathrm{~g}$ and Supplementary Data 5). Two hundred eighty-four (hg.t., $P<9.874 \mathrm{E}-25$ ) and 731 (hg.t., $P<4.460 \mathrm{E}-34$ ) deregulated genes were directly bound by HLX in K562 and HL60 cells, respectively (Supplementary Data 4). Expression of some ETC genes bound by HLX was increased, whereas that of PPAR $\delta$ was decreased, consistent with our results in zebrafish (Fig. 4h). Additionally, we performed qPCR for ETC and PPAR $\delta$ genes in K562 cells stably overexpressing an inducible $H L X$ construct. These experiments showed that increased HLX expression leads to high PPAR $\delta$ expression with concomitant lower expression of ETC genes (Supplementary Fig. 5g).

Here, we show that HLX directly regulates ETC and PPAR $\delta$ gene transcription, and that this function is conserved from zebrafish to humans. These exciting results suggest that HLX controls myeloid differentiation, at least partly, through metabolic

Fig. $2 \mathrm{hl} / \mathrm{x}$ regulates the transcription of metabolic genes. a Heatmap of z-transformed normalized gene expression values from RNA-Seq performed on sorted endothelial/hematopoietic (fli:kaede ${ }^{+}$) cells from fli:hHLXOE embryos or endothelial cells (kdrl:GFP+) from hlx1MO at 48 hpf after unsupervised hierarchical clustering with Euclidean distance metric (see also Supplementary Data 1). b IPA analysis of deregulated genes in the RNA-Seq data from fli: $\mathrm{hHLXOE}$ or hlx $1 \mathrm{MO}$ embryos at $48 \mathrm{hpf}$ (NBT, $P \leq 0.05$ and $\geq 2$ fold change) (also see Supplementary Data 1). c Mean density plots of read distribution of lost and gained ATAC-Seq peaks between control and hlx1MO (see also Supplementary Data 2). d Digital genomic footprinting analysis showing average normalized Tn5 insertion profiles around footprinted motifs in merged ATAC peaks as indicated for control and h/x $1 \mathrm{MO}$ (co-occurrence enrichment computation, $z=3.583$ and $z=13.241$, respectively). Insertions on the forward and reverse strands are indicated in red and blue, respectively. The numbers of motifs are indicated at the bottom of each panel. e Representative gene tracks from ATAC-Seq data of ETC and ppardb genes. $\mathbf{f}$ Heatmap of ETC gene expression in hlx1MO or fli:hHLXOE compared to control from the RNA-Seq analysis. All represented genes show differential chromatin accessibility in ATAC-Seq. $\mathbf{g}-\mathbf{i}$ qPCR analysis of selected ppar genes in $\mathbf{g}$ endothelial cells from $h / x 1 \mathrm{MO}$ (data representative of two independant experiments, mean + s.d.) or (h) whole fli:hHLXOE embryos at $48 \mathrm{hpf}\left(n=3\right.$, mean + s.d., Student's $t$-test, $\left.{ }^{\star} P<0.05\right)$ or (i) whole Runx:hHLXOE embryos at 5 dpf $(n=2$, mean + s.d.) 
a

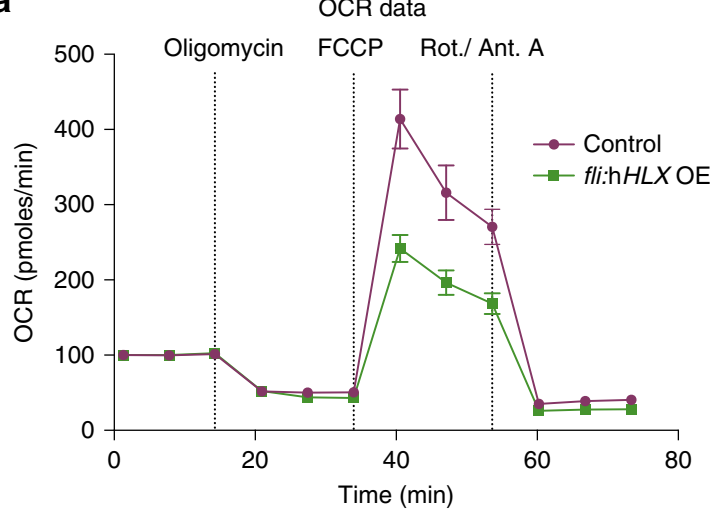

b
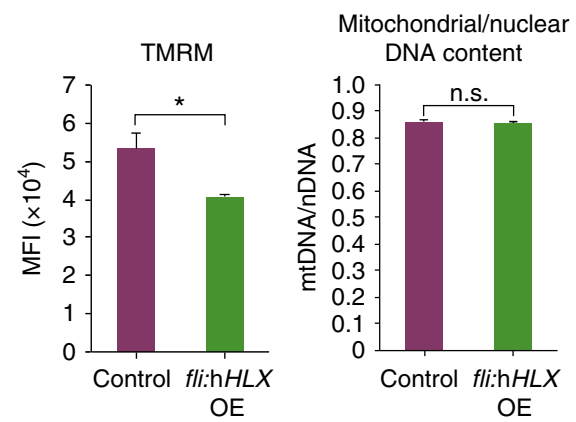

C

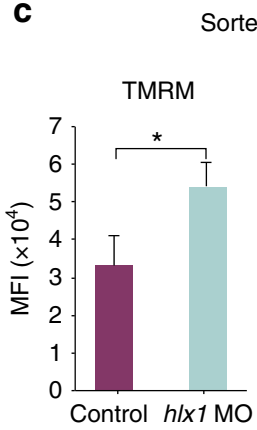

d Mitochondrial/nuclear

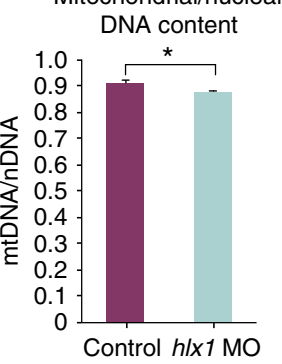

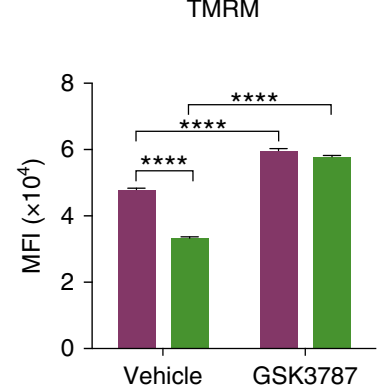

Mitochondrial/nuclear DNA content e

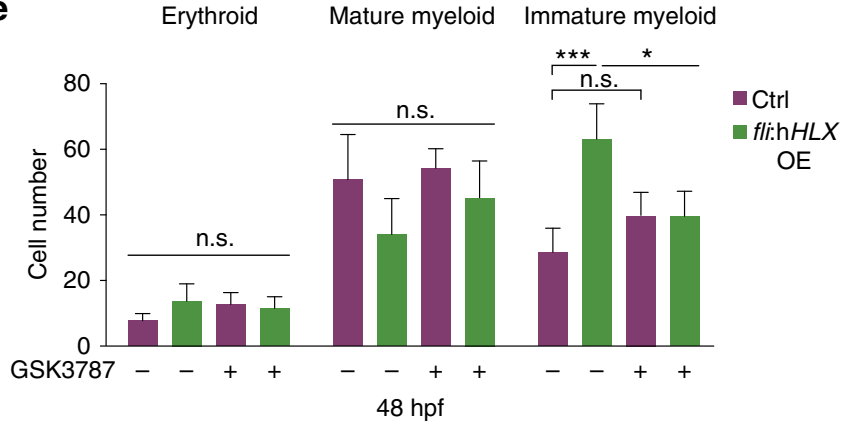

f

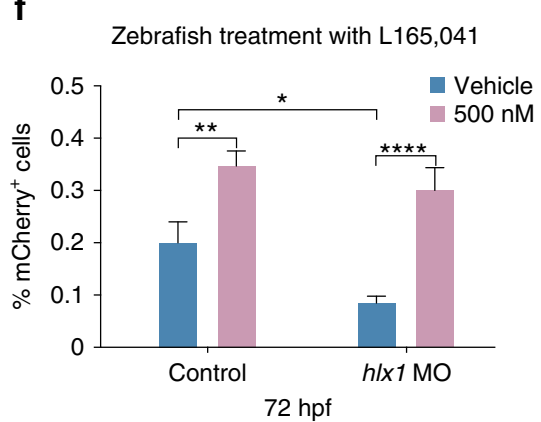

Fig. 3 PPARס modulation can rescue HSPC formation and myeloid differentiation in zebrafish. a Oxygen consumption rate (OCR) in control or fli:hHLXOE zebrafish. Representative plot from three independent experiments (mean \pm s.d.). b-c Mitochondrial membrane potential measured by TMRM stain (left panel) and mitochondrial/nuclear DNA content analysis (right panel) in (b) control and fli:hHLXOE embryos or in (c) endothelial/hematopoietic cells (fli: GFP positive) of control and $h / x 1 M O$ embryos at $48 \mathrm{hpf}\left(n=3\right.$, mean + s.d., Student's $t$-test, $\left.{ }^{\star} P<0.05\right)$. Graphs for TMRM depict median fluorescence intensity (MFI). The ratio of mitochondrial DNA (mtDNA) vs. nuclear DNA (nDNA) was measured at the same time $(n=3$, mean + s.d., Student's $t$-test, ${ }^{\star} P<0.05$, n.s. non-significant). d Mitochondrial membrane potential at $48 \mathrm{hpf}$ (left panel) in control or fli:hHLXOE embryos after GSK3787 treatment and corresponding ratio of mtDNA/nDNA (right panel) $\left(n=3\right.$, mean + s.d., ANOVA test, ${ }^{\star \star} P<0.01, \star \star \star P<0.001$, $\left.{ }^{\star \star \star \star} P<0.0001\right)$. e Cell count of zebrafish CHT smears from control or fli:hHLXOE embryos at $48 \mathrm{hpf}$ after treatment with GSK3787 $\left(n=4\right.$, mean + s.d., Student's $t$-test, ${ }^{\star} P<0.05$, $\left.{ }^{\star \star \star} P<0.001\right)$. f Percentage of Runx:mCherry HSPCs in Tg(Runx:mCherry) control or hlx1MO embryos at $72 \mathrm{hpf}$ after L165,041 (PPAR $\delta$ agonist) treatment ( $n=3$, mean + s.d., ANOVA test, $\left.{ }^{\star} P<0.05,{ }^{\star \star} P<0.01,{ }^{\star \star \star \star} P<0.0001\right)$

regulation. The combination of high PPAR $\delta$ expression with low respiratory chain activity resembles the effects of pathways involved in controlling LT-HSC stemness $28,29,32$ and could explain why $H L X$-overexpressing cells fail to terminally differentiate.

HLX overexpression leads to elevated AMPK and autophagy. HLX is particularly highly expressed in M4 and M5 AML leukemias $^{21}$. We therefore selected THP1 cells deriving from a patient with AML (M5 subtype) to further explore the metabolic function of HLX. ChIP in HLX-overexpressing THP1 cells revealed that HLX binds to 5827 genes (Supplementary Data 4).
HLX was often found bound to intronic (42.24\%) and intragenic $(44.7 \%)$ regions, and specifically to ETC and PPAR $\delta$ genes (Fig. 5a). HLX peaks were enriched for H3K27ac, a marker of active enhancers and promoters (Fig. 5b). Comparison of HLX ChIP in K562, HL60, and THP1 cells showed that 745 (12.8\%, hg.t., $P<7.838 \mathrm{E}-46)$ and 1767 (30\%, hg.t., $P<6.676 \mathrm{E}-18)$ of genes bound by HLX in THP1 cells were also bound in K562 and HL60 cells, respectively. To investigate whether the bound regions of HLX in THP1 cells represent open chromatin regions in HSCs and/or preleukemic and leukemic HSCs of AML patients, we used publicly available ATAC-Seq data ${ }^{39}$ and selected randomly one donor for each condition. Strikingly, all HLX-bound regions fall into accessible chromatin regions in HSCs, pre-leukemic, and 
a

\begin{tabular}{lc} 
Ingenuity analysis: K562 & \\
\hline Name & $P$-value \\
\hline Molecular Mechanism of Cancer & $3.76 \mathrm{E}-06$ \\
PPAR $\alpha /$ RXRActivation & $1.98 \mathrm{E}-05$ \\
TGF- $\beta$ Signaling & $3.77 \mathrm{E}-05$ \\
Decreases Transmembrane Potential of & \\
Mitochondria and Mitochondrial Membrane & $9.53 \mathrm{E}-03$ \\
Ingenuity analysis: HL-60 & \\
Name & $P$-value \\
\hline Molecular Mechanism of Cancer & $1.89 \mathrm{E}-05$ \\
Increases Transmembrane Potential of & \\
Mitochondria and Mitochondrial Membrane & $9.87 \mathrm{E}-05$ \\
TR/RXR Activation & $4.37 \mathrm{E}-04$ \\
PPAR $\alpha /$ RXRActivation & $3.90 \mathrm{E}-03$
\end{tabular}

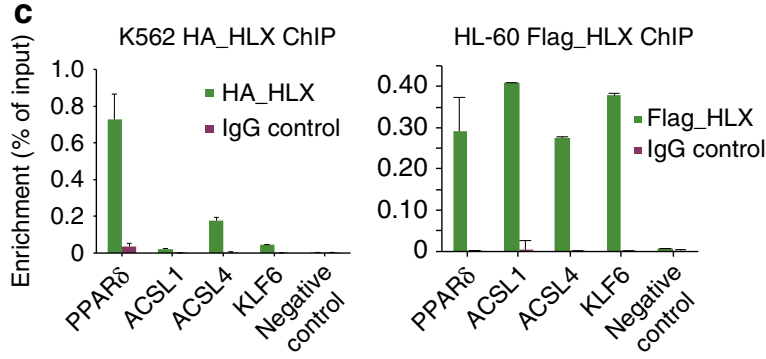

d

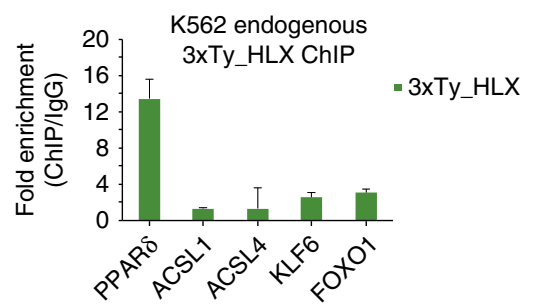

b
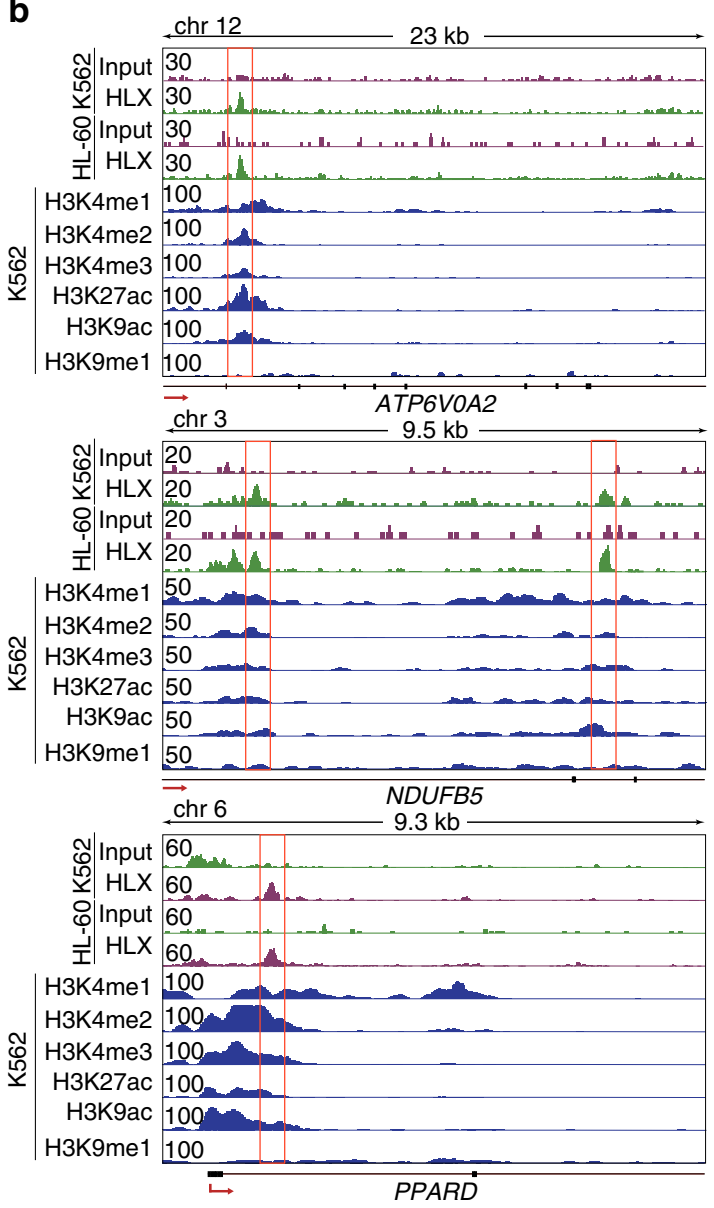

e

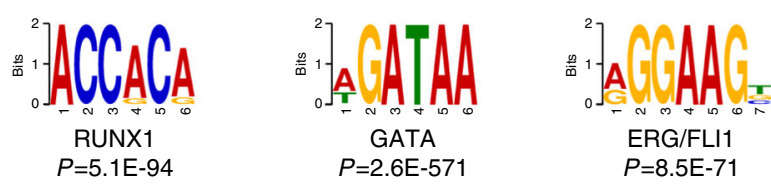

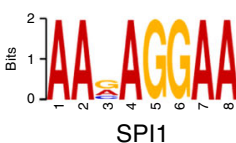

$P=5.9 \mathrm{E}-16$

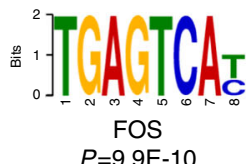

$P=9.9 \mathrm{E}-10$
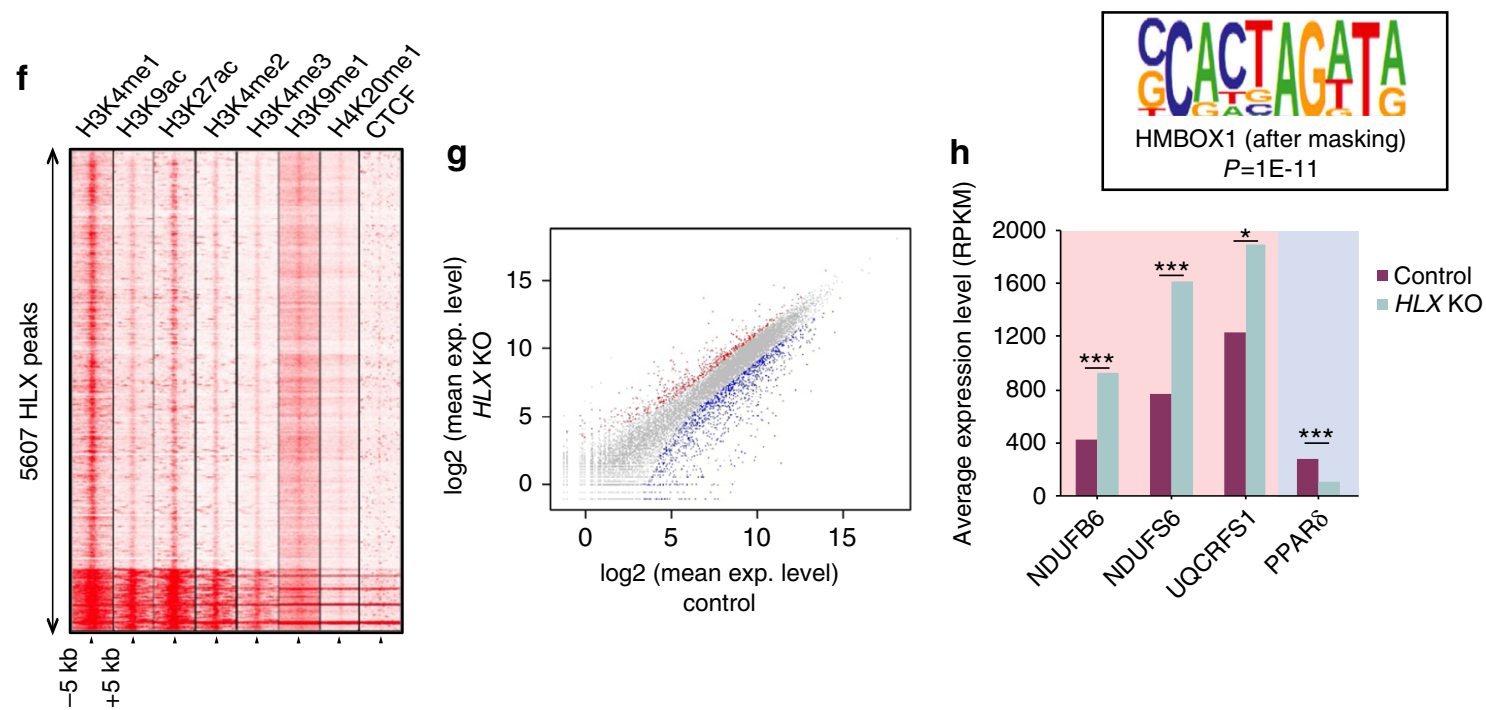

g

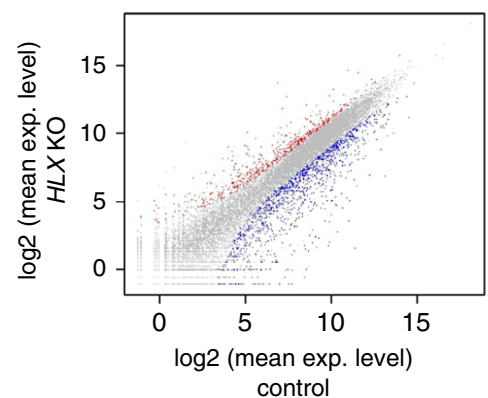

Control $\mathrm{HLXKO}$

leukemic HSCs (Fig. 5c). Thus, it is possible that perturbation of HLX binding affects genes implicated in HSCs or leukemic transformation.

Consistent with our previous results, ETC genes were downregulated and PPAR $\delta$ was upregulated at the protein level in
THP1 cells (Fig. 5d). Moreover, maximal respiratory capacity was reduced, and lower levels of reactive oxygen species (ROS) were produced, in HLX-overexpressing THP1 cells (Fig. 5e, f). As PPAR $\delta$ is a well-established regulator of fatty acid metabolism, we performed carbon tracing in control and HLX-overexpressing 
THP1 cells cultured with ${ }^{13} \mathrm{C}$-glucose. Indeed, we found increased incorporation of glucose-derived carbon in citric acid that can be used to produce fatty acids, which was also reflected in palmitic acid, fatty acid C18, and stearic acid (Supplementary Fig. 6).

To uncover the mechanisms downstream of ETC gene downregulation by HLX, we took a candidate approach and examined the AMPK pathway. It has recently been proposed that mitochondrial dysfunction can lead to AMPK activation ${ }^{40}$. Moreover, AMPK is a well-established sensor of metabolic stress and its activation results in elevated autophagy ${ }^{41,42}$. We found that AMPKa and phospho-AMPKa (p-AMPKa) are upregulated in HLX-overexpressing THP1 cells (Fig. 5d). Additionally, the protein levels of LC3-II, an autophagosome marker, are markedly increased in these cells upon chloroquine treatment (Fig. 5g).

Together, these results suggest that HLX overexpression in AML cells affects mitochondrial metabolism and fatty acid synthesis possibly through the upregulation of $P P A R \delta$ gene expression. Additionally, HLX overexpression, possibly through downregulation of ETC genes, results in AMPK activation and autophagy.

HLX regulates the metabolic state of $\mathrm{CD}^{+} 4^{+}$human cells. Our findings could have important implications for patients with hematopoietic disorders, including AML. We therefore performed colony-forming unit (CFU) assays on human $\mathrm{CD} 34^{+}$ HSPCs to measure the effects of HLX modulation in normal hematopoiesis. Consistent with our zebrafish results and with published data on mouse HSPCs ${ }^{21}$, HLX knockdown (sh-HLX) caused a significant reduction in the number of hematopoietic colonies, whereas HLX overexpression $\left(\mathrm{CD} 34^{+} H L X\right)$ caused the opposite phenotype and large myeloid colonies (Fig. 6a, b and Supplementary Fig. 7a). Culturing CD34 ${ }^{+} H L X$ in myeloid differentiation media resulted in a maturation block, as revealed by the accumulation of early granulocyte-monocyte progenitors (early GMPs) and the relatively reduced numbers of mature monocytes and granulocytes (Fig. 6c and Supplementary Fig. 7b). To assess whether PPAR $\delta$ and ETC genes are regulated by HLX in CD34 ${ }^{+}$cells, we performed RNA-Seq experiments upon $H L X$ knockdown or overexpression. Due to the high variability in primary cells we considered all the genes that have at least 1.5fold change independently of $P$-value. The expression of many ETC genes was clearly upregulated upon sh-HLX and downregulated in $\mathrm{CD}_{3}{ }^{+} H L X$ cells (Fig. 6d and Supplementary Data 5). Selected targets were validated with qPCR (Supplementary Fig. 7c). Thus, the function of HLX in hematopoiesis and its target genes are conserved in human primary hematopoietic cells.

To further determine whether the metabolic function of HLX is conserved in $\mathrm{CD}_{3}{ }^{+}$cells, we measured OXPHOS. Similar to zebrafish, increased levels of $H L X$ in human CD34+ cells led to a reduction in OCR, particularly spare respiratory capacity (Fig. 6e). Moreover, although $H L X$ overexpression did not cause significant changes on the OXPHOS to extracellular acidification rate (ECAR, representative of glycolytic rate) ratio, $H L X$-overexpressing cells tended to have a lower ratio (Supplementary Fig. 7d), suggesting a metabolic adaptation by engagement of glycolysis. TMRM staining shows that $\mathrm{CD} 34^{+} H L X$ cells have decreased mitochondrial membrane potential, independently of mitochondrial mass (Fig. 6f). However, upon differentiation toward myeloid cells, CD34 ${ }^{+} H L X$ cells exhibited significantly higher OXPHOS than control cells and a tendency to higher OCR/ECAR ratio (Supplementary Fig. 7e, f).

Our results in THP1 cells showed that HLX overexpression is followed by AMPK activation. To determine whether AMPK activation affects myeloid maturation, we induced differentiation of human $\mathrm{CD}_{3} 4^{+}$HSPCs in the presence or absence of metformin, an AMPK activator that also blocks mitochondrial complex I thus mimicking the effect of $\mathrm{HLX}^{43}$. Notably, metformin induced a myeloid differentiation block in $\mathrm{CD} 34^{+}$ cells (Fig. 6g). These results suggest that metabolic manipulation can indeed be the underlying reason for the hematopoietic phenotypes caused by HLX deregulation.

We next assessed whether pharmacological modulation of PPAR $\delta$ activity rescues the myeloid differentiation phenotypes caused by HLX. Indeed, treatment with a PPAR $\delta$ antagonist relieved the myeloid differentiation block in $\mathrm{CD}_{3}{ }^{+} H L X$ cells (Fig. 7a). Moreover, the inability of sh-HLX cells to form colonies in CFU assays was partially rescued by a PPAR $\delta$ agonist (L165,041) (Fig. 7b). This agonist also rescued the increased mitochondrial membrane potential observed in sh-HLX cells (Fig. 7c). To investigate this further, we identified genes affected by HLX and potentially regulated by PPAR $\delta$ using publicly available PPAR $\delta$-binding data in human macrophages ${ }^{44}$. We compared these data to our RNA-Seq from human $\mathrm{CD}^{+}{ }^{+}$cells and found that 399 (hg.t., $P<0.006$ ) deregulated genes upon $H L X$ knockdown that can potentially be bound directly by PPAR $\delta$ (Supplementary Data 6). IPA analysis on these genes showed involvement in FAO I (rtFET, $P<2.26$ E-04) and AMPK signaling (rtFET, $P<5.24$ E-03) (Supplementary Data 6).

AMPK inhibition causes reduced viability of AML cell lines. To investigate the potential role of PPAR $\delta$ and AMPK in promoting AML downstream of HLX, first, we analyzed the expression levels of HLX and PPAR $\delta$ by qPCR in various AML cell lines and one CML cell line, K562. As expected, THP1, a M5 subtype leukemia, exhibited the highest levels of HLX expression ${ }^{21}$ but also PPAR $\delta$ expression (Fig. 8a). PPAR $\delta$ protein was only detectable in THP1 cells (Fig. 8b). AMPKa and pAMPKa expression was detected in all cell lines without any noticeable differences (Fig. 8b). TMRM and autophagy were variable between cell lines (Fig. 8c, d). Next, we asked whether PPAR $\delta$ and AMPK inhibitors could push the AML lines and/or K562 cells toward myeloid maturation or affect their viability. PPAR $\delta$ antagonists (GSK3787) had no significant impact on either the viability or the differentiation of AML cell lines and K562 cells (Fig. 8e). However, AMPK inhibition with dorsomorphin significantly reduced the viability of all but one (HL60) AML cell lines tested and K562 cells (Fig. 8f). It is important to note that dorsomorphin had only a mild effect on the viability of $\mathrm{CD}^{+} 4^{+}$myeloid progenitor cell populations (Fig. 8f).

Fig. 4 Direct binding of HLX on metabolic genes. a IPA analysis of K562 and HL60 HLX ChIP-Seq peaks ( $P$-values rtFET, see also Supplementary Data 4 ). b Representative gene tracks of HLX-bound genomic regions in K562 and HL60 cells, together with publicly available data for histone modifications in K562 cells. c ChIP-qPCR validation of selected HLX peaks in K562 and HL60 cells $(n=2$, mean + s.d.). d ChIP-qPCR validation upon ChIP of the endogenous 3xTy-tagged HLX in K562 cells ( $n=2$, mean + s.d.). e Representative motifs identified in HLX-bound genomic regions in K562 cells ( $P$-values binomial test, see also Supplementary Data 4). HOX motif is shown in the frame. $\mathbf{f}$ Heatmap of ChIP-Seq signals, comparing HLX-bound regions in K562 cells to publicly available CHIP-Seq data for histone marks in K562 cells from the ENCODE database. CTCF was used as negative control. $\mathbf{g}$ Gene expression in control samples plotted vs. gene expression in HLX KO K562 cells. Differentially expressed genes ( $>2$-fold, NBT, $P<0.05)$ are colored in red and blue for upregulated and downregulated genes, respectively (also see Supplementary Data 5). h Average expression levels of selected genes from RNA-Seq of K562 HLX KO cell line (Student's $t$-test, ${ }^{\star} P<0.05,{ }^{\star \star \star} P<0.001$ ) 
a
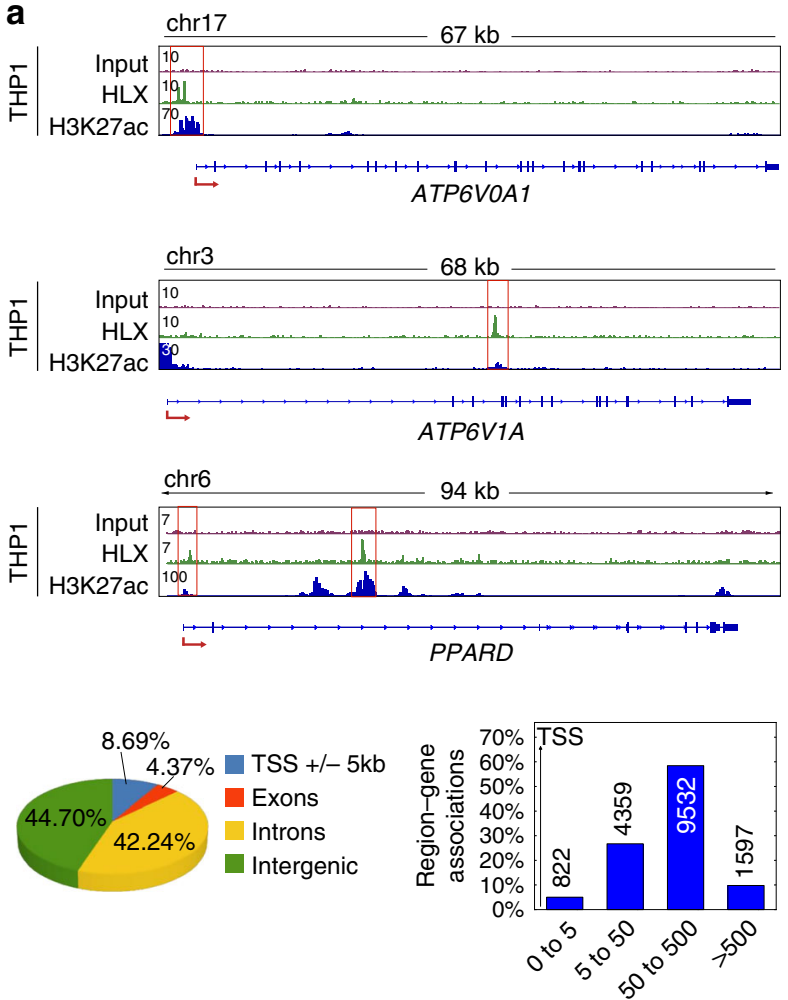

Absolute distance to TSS $(\mathrm{kb})$ b

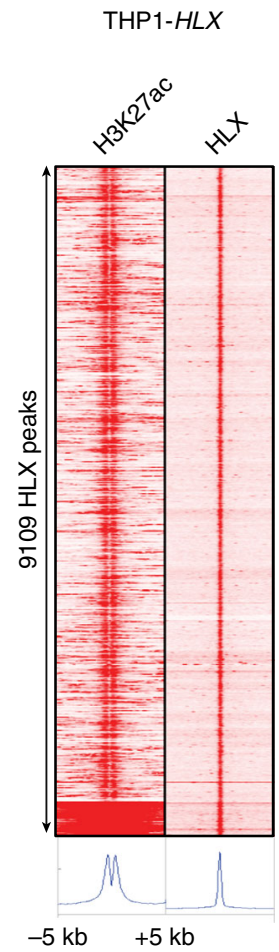

C

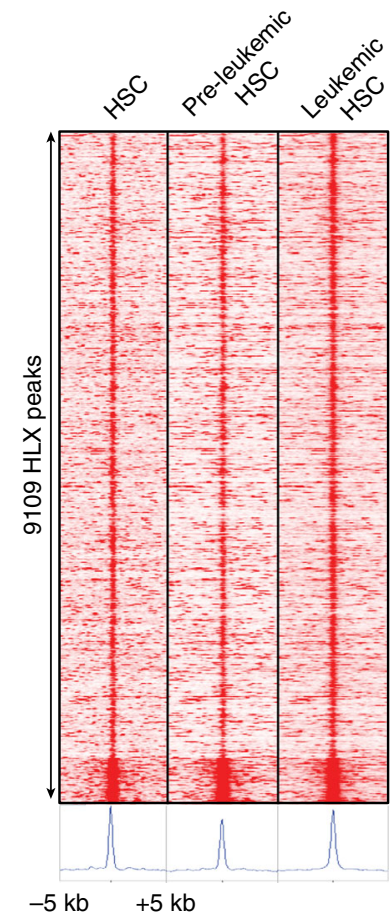

d

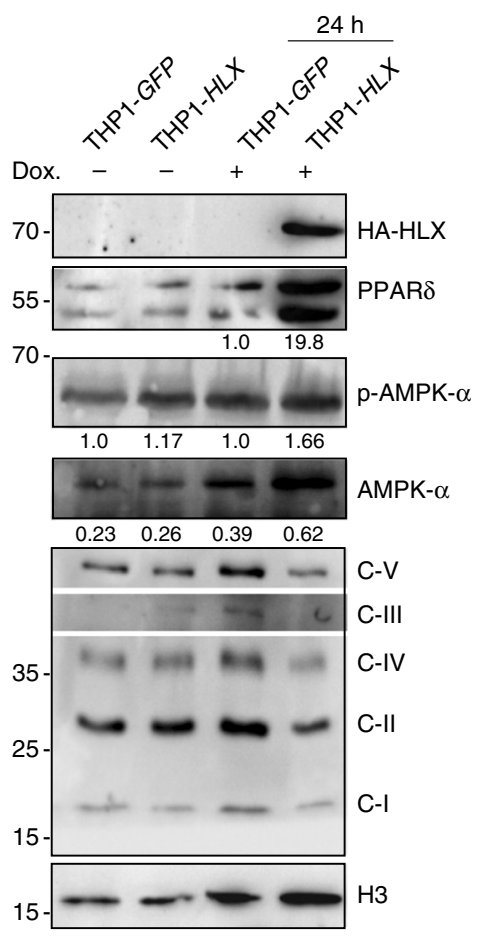

e

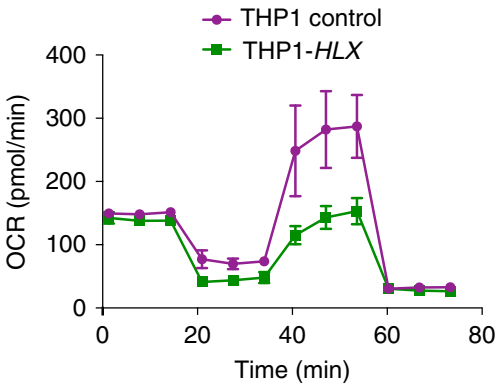

9

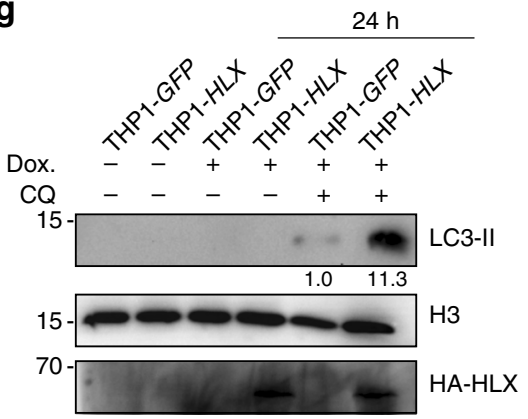

f 
was recently shown by Huang et al. that HLX controls a systematic switch from white to brown fat through metabolic gene regulation, including PPARs and genes that control mitochondrial biogenesis ${ }^{24}$. However, in that study HLX was found to positively regulate both mitochondrial biogenesis and PPARs, in contrast to our study where ETC genes are downregulated upon HLX overexpression. It will be interesting to study whether diverse HLX-interacting partners could account for these differences.

Recently, metabolism has emerged as a critical regulator of HSCs. LT-HSCs are quiescent and rely mostly on anaerobic glycolysis rather than OXPHOS ${ }^{47,48}$. A number of studies have shown that low mitochondrial activity is necessary to maintain the quiescent state and the self-renewal capacity of LT-HSCs and protect them from oxidative stress ${ }^{28,49-53}$. The importance of reduced mitochondrial activity for HSC maintenance has also been demonstrated in human $\mathrm{CD}_{3}{ }^{+} \mathrm{HSPCs}^{54-56}$. Antagonism of PPAR $\gamma$ signaling enhances glycolysis and leads to expansion of human $\mathrm{HSPCs}^{57}$. Moreover, recent evidence revealed that PPAR $\delta$, a regulator of fatty acid metabolism, plays an essential role in maintaining HSC stemness by regulating mitophagy and promoting HSC asymmetric cell divisions ${ }^{32,58}$. During differentiation, PPAR $\delta$ signaling is downregulated leading to a gradual increase in mitochondrial mass and symmetric commitment of HSC daughter cells ${ }^{32}$. Thus, PPAR $\delta$, but also HLX that controls its expression, may constitute a metabolic switch for regulating HSC cell fate. It is interesting to speculate, based on our results, that HLX is a gatekeeper of HSC identity by maintaining their glycolytic state. We also show that HLX overexpression leads to low spare respiratory capacity, a characteristic of AML cells ${ }^{59}$. Both these results could be used in the future to better understand the implication of HLX in AML. However, since both HLX and $\operatorname{PPAR} \delta$ exert many functions besides metabolic regulation, we need to fully understand the precise mechanisms underlying our rescue experiments by PPAR $\delta$ modulation. Interestingly, since PPAR $\delta$, like HLX, is overexpressed in a subset of M5 typemonoblastic AML cases ${ }^{60}$, it is conceivable that PPAR $\delta$ inhibition could play a role in AML. Our results do not support this hypothesis, but further investigations should shed more light on a potential role of PPAR $\delta$ in AML.

Also of note, we show that metformin, which blocks mitochondrial complex I and activates $\mathrm{AMPK}^{43}$, thereby mimicking the metabolic function of HLX, can affect myeloid differentiation. This finding proves that metabolic regulation can indeed be the direct mediator of HLX functions and, at least in part, causative for the phenotype. Metformin has been used as an anticancer therapy in many malignancies, including $\mathrm{AML}^{61}$. Based on our findings, it is pertinent to fully understand the role of metformin in physiological and pathological conditions.

Finally, we found that HLX overexpression leads to activation of AMPK. AMPK does not seem to play a role in HSCs during homeostasis, transplantation, or under metabolic stress ${ }^{62}$, but protects leukemia initiating cells from metabolic stress ${ }^{63}$. This suggests AMPK as an ideal potential target for the treatment of leukemia without affecting normal cells. Indeed, we found that AMPK inhibition has a strong effect on the survival of AML cell lines. AMPK inhibition is also successful in eliminating MLLrearranged B-cell acute lymphoblastic leukemia ${ }^{64}$. However, other studies suggest that AMPK acts synergistically with mTORC1 and causes lethality in AML cells ${ }^{65}$. Further research with samples from human patients and specific mouse models are needed to clarify these discrepancies.

Our study points to differential requirements and regulatory mechanisms between normal and leukemic cells by the same transcription factor and identifies HLX as a new player in metabolic regulation in hematopoiesis.

\section{Methods}

Zebrafish maintenance. The zebrafish (D. rerio) strain Tübingen (Tü) and all zebrafish transgenic lines used in this study were maintained in the animal facility of the Max Planck Institute of Immunobiology and Epigenetics. The sample size for the animal experiments was chosen according to the following paper ${ }^{66}$. No animals were excluded from this study and no randomization was used. Only 1-5 dpf embryos were used in this study and sex was not determined at these stages. All animal experiments were performed in accordance with relevant guidelines and regulations, approved by the review committee of the Max Planck Institute of Immunobiology and Epigenetics and the Regierungspräsidium Freiburg, Germany (license Az 35-9185.81/G-14/95)

Zebrafish morpholino injections and rescue experiments. Embryos were injected (PV820 Pneumatic PicoPump, World Precision Instruments) at the onecell stage with $8 \mathrm{ng}$ of standard morpholino or $8 \mathrm{ng}$ of $h l x 1$ translational or $12 \mathrm{ng}$ splicing anti-sense morpholino that have been previously described ${ }^{26}$ (Gene Tools, Philomath, OR). Stock solutions were diluted as recommended by the manufacturer. The sequence for the $h l x 1$ translational anti-sense morpholino: $5^{\prime}$ AGCCGAACAATACGCAGTCCACAGG-3'; splicing anti-sense morpholino: 5' GATTAAATTAGCGTCTTACCTCTCA-3'; standard oligo: 5' -CCTCTTACCT CAGTTACAATTTATA-3'

For the rescue experiments one-cell stage $\operatorname{Tg}(\text { Runx:mCherry })^{27}$ embryos were injected with 12.5 or $25 \mathrm{pg}$ of fli:HLX or Runx:HLX constructs followed by injection of $h l x 1$ morpholino as described above. Injected embryos were grown until $72 \mathrm{hpf}$ manually dechorionated and embedded in $1 \%$ low melting agarose, containing $0.04 \mathrm{mg} / \mathrm{mL}$ tricaine. Caudal hematopoietic tissue was imaged using Zeiss Apotome 2 microscope at $10 \times$ magnification. Flow cytometry of mCherry-positive cells is described in the section "Preparation of zebrafish cells, flow cytometry, and cell sorting". WISH staining and analysis, constructs and generation of transgenic zebrafish lines are described in Supplementary Material and Methods.

Preparation of zebrafish cells, flow cytometry, and cell sorting. Embryos were incubated in $0.5 \mathrm{mg} / \mathrm{mL}$ Liberase TM (Roche) solution for $30 \mathrm{~min}$ at $37^{\circ} \mathrm{C}$, then dissociated and resuspended in PBS-5\% fetal bovine serum (FBS), and used for cell sorting, flow cytometry, seahorse assay, qPCR, and RNA-Seq experiments. Cell sorting was performed using Influx (BD Biosciences). For all experiments cellsorting purity was over $85 \%$.

May-Grünwald-Giemsa staining of zebrafish blood. For CHT smears, $48 \mathrm{hpf}$ (fli:hHLXOE) or $5 \mathrm{dpf}$ (Runx:hHLXOE) embryos were placed in $0.9 \% \mathrm{NaCl}$ with $0.04 \mathrm{mg} / \mathrm{mL}$ tricaine and the tails were isolated at the level of the cloaca/end of the yolk sac extension and incubated with Liberase TM (at 1:65 dilution in $0.9 \% \mathrm{NaCl}$, Roche) with $0.04 \mathrm{mg} / \mathrm{mL}$ tricaine for $20 \mathrm{~min}$ at $37^{\circ} \mathrm{C}$. FBS was then added to a final concentration of $10 \%$, to stop enzymatic digestion. The tails were triturated and then passed through a $40 \mu \mathrm{M}$ mesh filter. The smears of dissociated cells were prepared by cytospin followed by May-Grünwald-Giemsa staining. Cells were imaged using Zeiss Axio Imager microscope with $100 \times$ objective.

Fig. 5 AMPK and autophagy activation in HLX-overexpressing cells. a Representative gene tracks of HLX and H3K27ac-bound genomic regions in THP1 cells. Location annotation of HLX-bound regions across the genome. $\mathbf{b}$ Heatmap of ChIP-Seq signals comparing HLX-bound regions to H3K27ac regions in THP1 cells. c Heatmap comparing HLX-bound regions in THP1 cells to ATAC-Seq regions of HSCs, pre-leukemic HSCs, and leukemic HSCs. d Western blot analysis of HA-HLX, PPARS, AMPK $\alpha$, and p-AMPK $\alpha$, mitochondrial ETC complexes and Histone $3(\mathrm{H} 3)$ as loading control. Cells were untreated or treated with doxycycline for $24 \mathrm{~h}$ to induce HLX expression. Representative immunoblots of at least three independent experiments. Quantifications were performed by FIJI software software and are shown below the blots as a ratio to H3. The five OXPHOS complexes are depicted as C-I to V. e Oxygen consumption rate (OCR) in control or HLX-overexpressing THP1 cells. Representative plot from three independent experiments (mean $\pm \mathrm{s}$.d.). $\mathbf{f}$ Bar graph depicting mitochondrial ROS production in control and THP1-HLX cells $\left(n=3\right.$, mean + s.d., Student's $t$-test, $\left.{ }^{\star \star} P<0.01\right)$. $\mathbf{g}$ Western blot analysis of LC3-II, HA, and H3 in control and THP1-HLX cells non-induced, induced with Doxycycline (Dox) with or without the addition of Chloroquine (CQ). Representative immunoblots of three independent experiments. Quantification was performed by Fiji software and is shown below the blot as a ratio to $\mathrm{H3}$ 
a

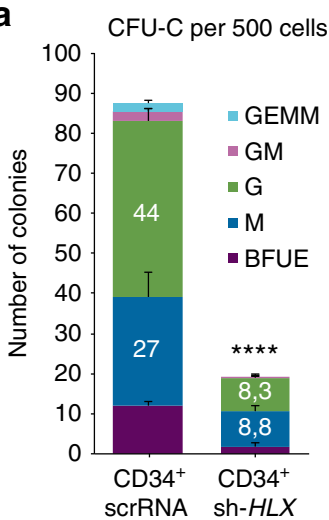

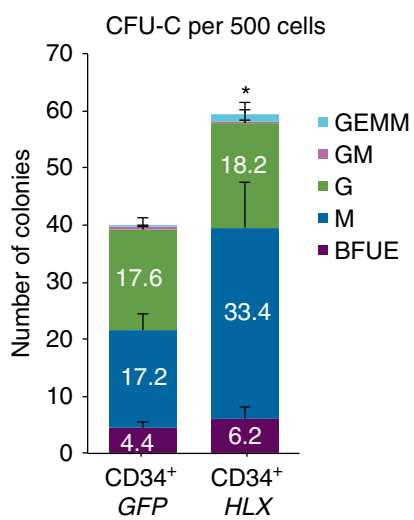

b

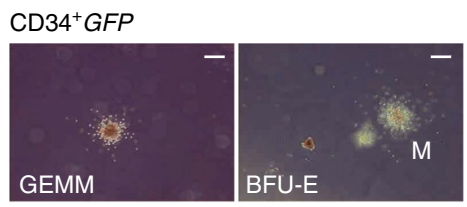

CD34 ${ }^{+} H L X$

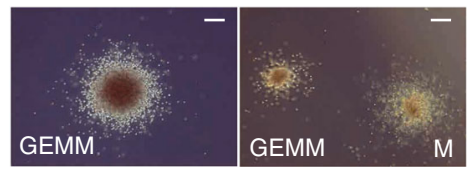

c

$\mathrm{CD}^{+}{ }^{+}$differentiation toward myeloid $(7 \mathrm{~d}$.
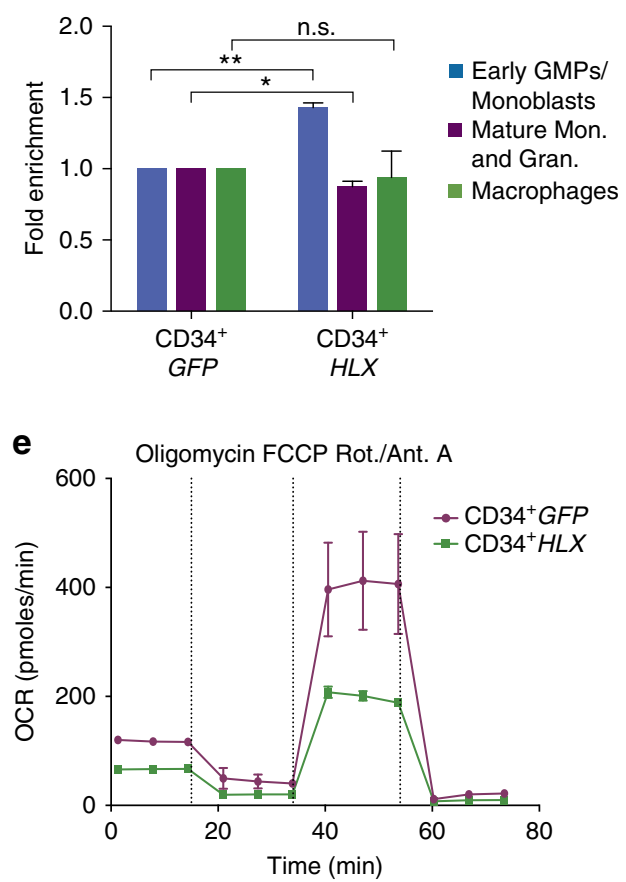

d
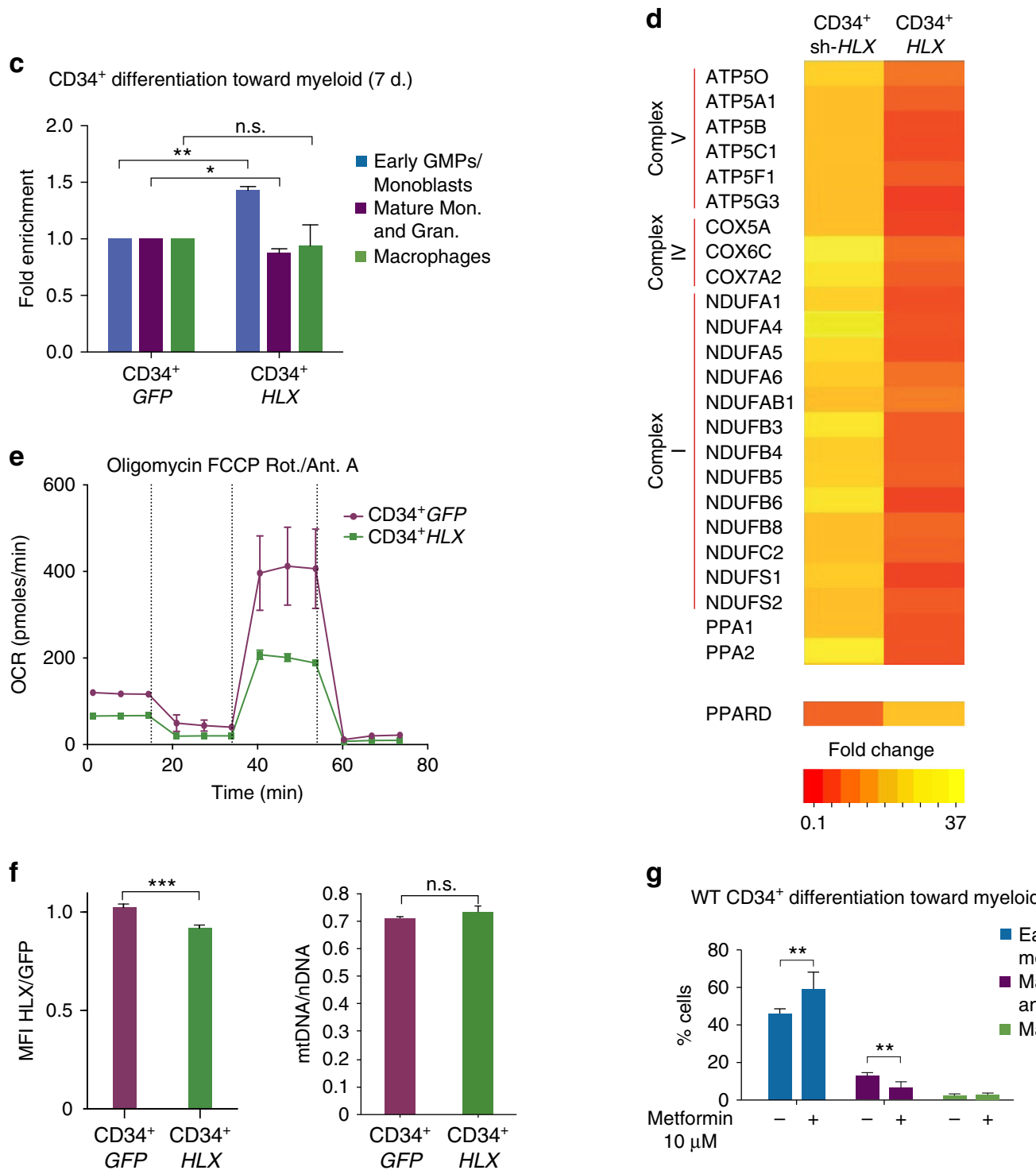

g

WT CD $34^{+}$differentiation toward myeloid $(7 \mathrm{~d}$.)

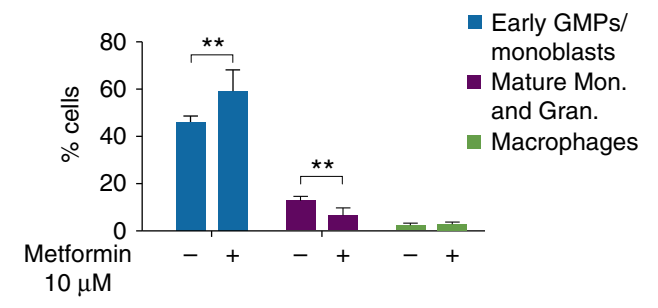

TUNEL assay. Whole-mount TUNEL staining of developmentally staged control, morphant, and overexpression embryos was performed using the in situ cell death detection kit with fluorescein (Roche Applied Science, 11684795910). Embryos were then embedded in $1 \%$ low melting agarose and imaged with a Zeiss LSM780 confocal microscope and a $10 \times$ objective.

EdU labeling. Cell proliferation in zebrafish was assayed using the Click-iT EdU Alexa Fluor 647 Imaging Kit (Thermo Fisher Scientific). Briefly, zebrafish embryos at $48 \mathrm{hpf}$ were dechorionated and incubated with Liberase TM as described in the section "Preparation of zebrafish cells, flow cytometry, and cell sorting".
Dissociated cells were spun down, resuspended in EdU staining solution, and incubated for $30 \mathrm{~min}$ at $37^{\circ} \mathrm{C}$. Cells were fixed and permeabilized, followed by EdU detection reaction, according to the manufacturers's instructions. Cells were then analyzed by flow cytometry (BD LSRFortessa).

Cell line and primary cell maintenance. K562 and SKNO1 cells were maintained in Iscove's Modified Dulbecco's Medium (IMDM, Gibco) supplemented with $10 \%$ FBS (Sigma, F7524) and 1\% Penicillin Streptomycin (P/S, $100 \mathrm{U} / \mathrm{mL}$ penicillin with $100 \mu \mathrm{g} / \mathrm{mL}$ streptomycin, Gibco). KG1 cells were maintained in IMDM 20\% FBS, $1 \% \mathrm{P} / \mathrm{S}$. HL60, NB4, and THP-1 cells were maintained in RPMI 1640 medium 
Fig. 6 Metabolic role of HLX in human $\mathrm{CD}_{4} 4^{+}$cells. a Average number of colonies in CFU-C assays performed in CD34 ${ }^{+}$cells infected with control scrRNA or sh-HLX (left panel) or in CD34+ cells overexpressing GFP (CD34+ GFP) or HLX (CD34+ HLX) (right panel) $(n=3$, three technical replicates each time, mean + s.d., Student's t-test, $\left.{ }^{\star} P<0.05,{ }^{\star \star \star \star} P<0.0001\right)$. GEMM Granulocyte, Erythrocyte, Monocyte, Megakaryocyte, GM Granulocyte, Macrophage, G Granulocyte, M Macrophage, BFUE Burst-forming unit-erythroid. b Representative images of GEMM, BFUE, and M colonies from CFU-C assays in CD34+ GFP or CD34+ HLX cells. Scale bar: $50 \mu \mathrm{M}$. c Flow cytometric analysis representing elevated numbers of immature myeloid cells in CD34+ ${ }^{+} \mathrm{LX}$ cells differentiated toward the myeloid lineage for 7 days $\left(n=3+\right.$ s.d., Student's $t$-test, $\left.{ }^{\star} P<0.05,{ }^{\star \star} P<0.01\right)$. $\mathbf{d}$ Heatmap representing the fold change of selected genes from RNA-Seq of $\mathrm{CD} 34^{+}$sh-HLX and CD34+ HLX cells compared to their respective controls. e Oxygen consumption rate in CD34+ GFP or $H L X$ cells before differentiation ( $n=3$, mean + s.d.). f Mitochondrial membrane potential measured by TMRM in CD34+ GFP or CD34+ HLX cells (left panel) $\left(n=6\right.$, mean + s.d., Student's t-test, $\left.{ }^{\star \star \star} P<0.001\right)$ and the corresponding mitochondrial vs. nuclear DNA ratio (right panel) $(n=3$, mean + s.d., Student's $t$-test n.s.). g Flow cytometric analysis representing numbers of immature myeloid cells upon addition of metformin to wild-type $\mathrm{CD} 4^{+}$cells cultured in myeloid differentiation media for 7 days $\left(n=3\right.$, mean + s.d., Student's $t$-test, $\left.{ }^{\star \star} P<0.01\right)$
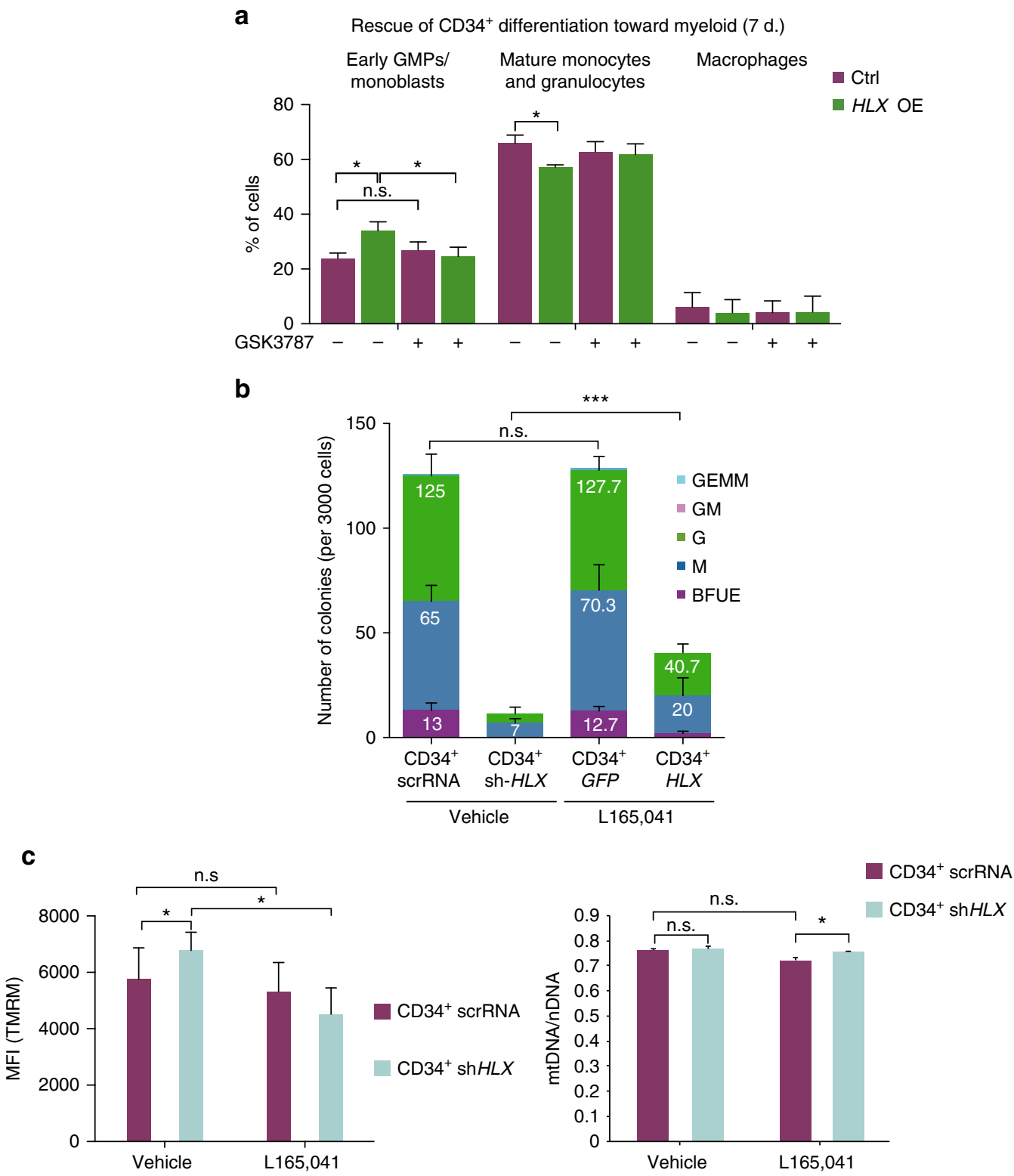

Fig. 7 PPARS modulation rescues hematopoietic phenotypes in human primary cells. a Flow cytometric analysis of CD34+ ${ }^{+}$FP or CD34+ $H L X$ cells cultured in differentiation media for 7 days in the presence or absence of the PPARS antagonist GSK3787 depicts rescue of the myeloid differentiation block after treatment $\left(n=3\right.$, Student's $t$-test, ${ }^{\star} P<0.05$, mean + s.d.). b Average number of colonies in CFU-C assays performed in CD34 ${ }^{+}$cells infected with control scrRNA or sh-HLX, treated or not with L165,041 $\left(n=2\right.$, three technical replicates each time, mean + s.d., Student's $t$-test, $\left.{ }^{\star \star \star} P<0.001\right)$. c Mitochondrial membrane potential measured by TMRM (left panel) and respective mitochondria vs. nuclear DNA ratio (right panel), in CD34 ${ }^{+}$infected with scrRNA or sh-HLX treated or not with $\mathrm{L} 165,041\left(n=3\right.$, mean + s.d., Student's $t$-test, $\left.{ }^{\star} P<0.05\right)$ 

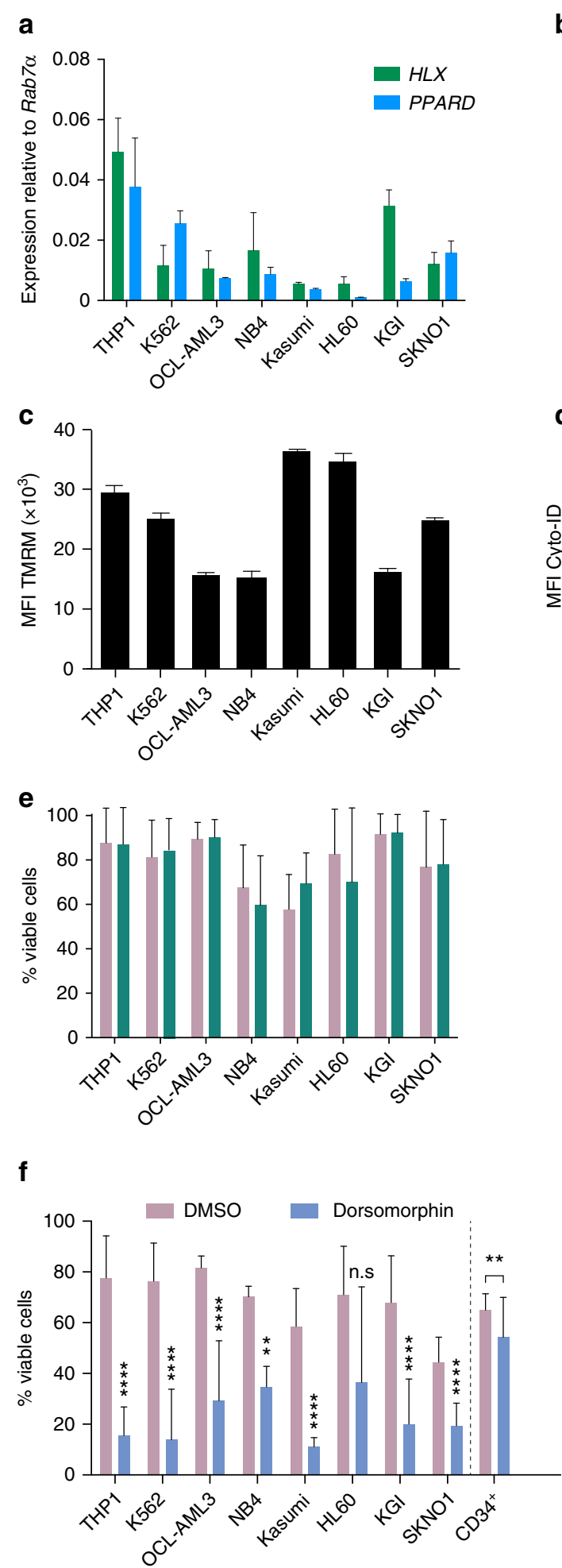

b

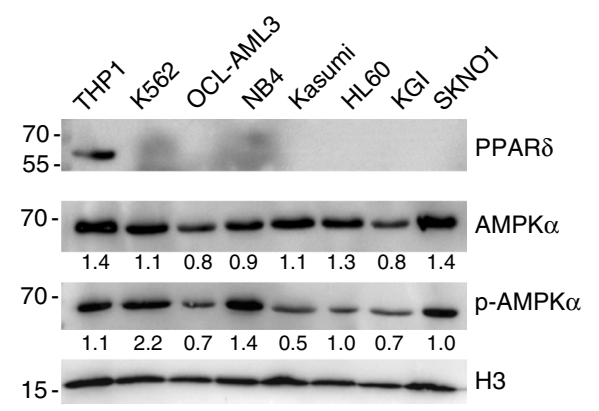

d
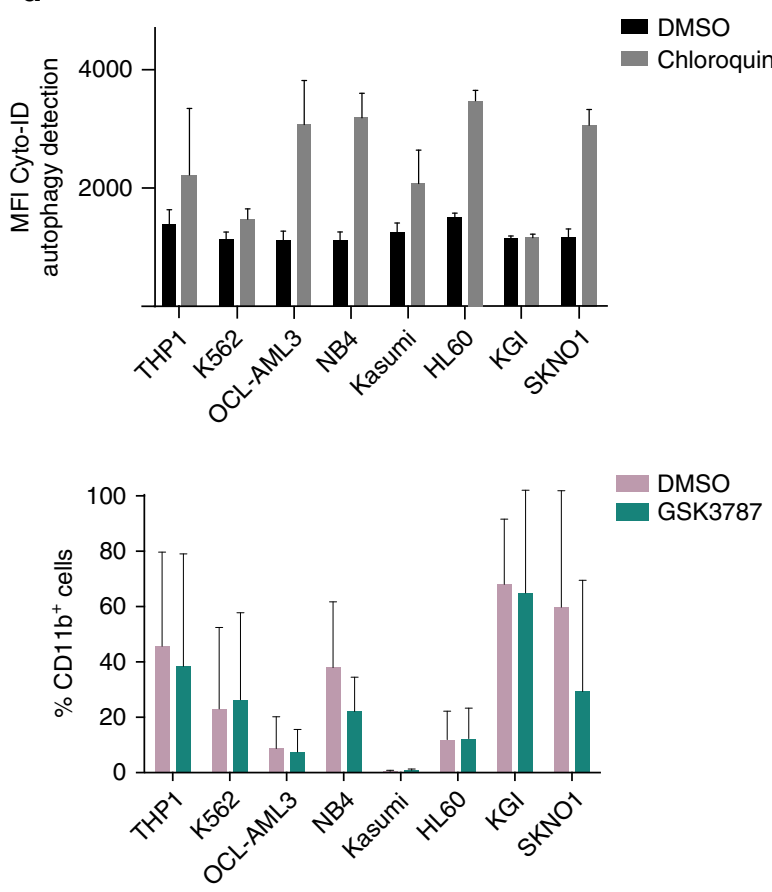

g

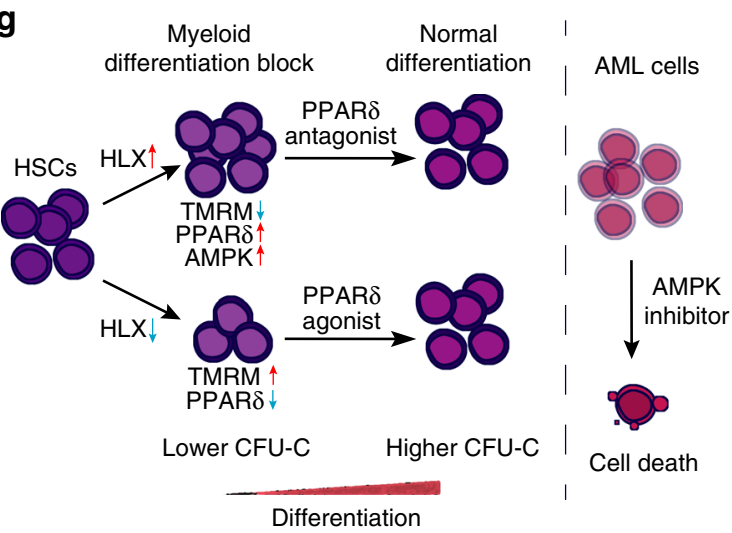

Fig. 8 AMPK inhibition causes lethality in AML cell lines. a qPCR expression analysis of $H L X$ and PPAR $\delta$ in AML cell lines and K562 cells ( $n=2$, mean + s.e.m.). b Representative western blot analysis for PPAR $(n=4), \operatorname{AMPK} \alpha(n=3), \mathrm{p}-\mathrm{AMPK} \alpha(n=2)$ and H3 as loading control in different AML cell lines and K562 cells. Quantifications shown below the panels, as a ratio to H3, were performed by FIJI software. c Mitochondrial membrane potential measured by TMRM in AML cell lines and K562 cells. MFI median fluorescence intensity (representative of two independent experiments, mean + s.d.). $\mathbf{d}$ Flow cytometry analysis of autophagic flux in different AML cell lines and K562 cells incubated with DMSO or chloroquine. MFI median fluorescence intensity (representative of two independent experiments, mean + s.d.). e Measurement of viable cells (left panel) or CD11b staining as a marker of myeloid differentiation (right panel) with or without the PPAR $\delta$ antagonist GSK3787 $(n=5$, mean + s.d.). f Measurement of viable cells after incubation of different AML cell lines and K562 cells or WT CD34+ cells with or without dorsomorphin $\left(n=3\right.$, mean + s.d., Student's $t$-test, $\left.{ }^{\star \star} P<0.01,{ }^{\star \star \star \star} P<0.0001\right)$. $\mathbf{g}$ Model of HLX function in hematopoiesis 
(Gibco) supplemented with $10 \%$ FBS, 1\% P/S. Kasumi cells in RPMI 1640 medium with $20 \% \mathrm{FBS}, 1 \% \mathrm{P} / \mathrm{S}$. The cell line OCI-AML3 was maintained in Alpha MEM (Gibco) supplemented with $20 \% \mathrm{FBS}, 1 \% \mathrm{P} / \mathrm{S}$. All media were supplemented with $2 \mu \mathrm{M}$ glutamine. K562 cells were verified from Eurofins and from RNA-Seq experiments and THP1 cells verified by next generation sequencing experiments. Cell lines were mycoplasma free as tested by qPCR. Cell lines were purchased from Sigma or ATCC or Lonza. Cell line generation and constructs can be found in the Supplementary Information.

Human $\mathrm{CD}_{3} 4^{+}$cells, isolated from the peripheral blood of granulocyte colonystimulating factor mobilized healthy volunteers, were purchased from the Fred Hutchinson Cancer Research Center. The cells were maintained as previously described ${ }^{67}$. Briefly cells were cultured in StemSpan SFEM (StemCell Technologies), supplemented with $2 \% \mathrm{P} / \mathrm{S}$ and cytokine mix: $\mathrm{m}-\mathrm{SCF} 100 \mathrm{ng} / \mathrm{mL}$, hFLT3 $100 \mathrm{ng} / \mathrm{mL}$, hIL-3 $20 \mathrm{ng} / \mathrm{mL}$, and hIL-6 $20 \mathrm{ng} / \mathrm{mL}$ (expansion medium). Cells were kept at $1 \times 10^{5}-1 \times 10^{6}$ cells $/ \mathrm{mL}$ densities.

Lentiviral particle production. $293 \mathrm{~T}$ cells were transfected with lentiviral plasmids (packaging vectors with GFP-HA or $H L X$-HA or sh-HLX or scrRNA) mix using the polyethylenimine (PEI) method. Briefly, $2 \mathrm{~h}$ before the transfection, fresh $2 \%$ FBS containing medium was added. For $9 \mathrm{~cm}$ culture dishes, $5 \mu \mathrm{g}$ of total DNA mix (construct, pPAX2, and pMDG.2 were used at a ratio of 10:7.5:3, respectively) was diluted with plain DMEM (Gibco) up to $520 \mu \mathrm{L} .30 \mu \mathrm{L}$ of $1 \mathrm{mg} / \mathrm{mL}$ PEI reagent was then added and the mix was vortexed shortly twice. The mix was incubated for $10 \mathrm{~min}$ at RT before adding drop wise to 293T cells. The medium was changed after $8 \mathrm{~h}$. Supernatants containing lentiviral particles were collected at 48,72 , and $96 \mathrm{~h}$ post transfection and concentrated using Lenti-X concentrator (TAKARA). All lentiviral experiments were performed in S2 laboratory with permission from German authorities: Regierungspräsidium Tübingen (57-3/8817.40-020).

Electroporation or infection of CD34 ${ }^{+}$cells. HLX overexpression: 1 day after thawing, 1 million cells were electroporated using the Human CD $34^{+}$Cell Nucleofector kit (Lonza) with $5 \mu \mathrm{g}$ pCMV6-AC-HLX (Origene, NM_021958) or as a control $5 \mu \mathrm{g}$ of pEGFP-C1 plasmids. A day after electroporation CD34 ${ }^{+}$GFP and $H L X$ cells were selected with neomycin $(0.8 \mathrm{mg} / \mathrm{mL})$ and expanded for 4 more days.

HLX knockdown: To generate shRNA against $H L X$ (sh-HLX), we cloned annealed oligos (Supplementary Table 1) into the pLKO.1-TCR vector (a gift from David Root; Addgene plasmid \#10878) digested with AgeI and EcoRI. As a control, a non-hairpin pLKO.1-TCR control (scrRNA) (a gift from David Root; Addgene plasmid $\# 10879)^{68}$ was used. For the generation of scrRNA and shHLX, human $\mathrm{CD}_{4}{ }^{+}$cells were infected after 1-day expansion in expansion medium (see section "Cell line and primary cell maintenance"). Cells were placed on retronectin-coated (TAKARA) plates and transduced with concentrated virus at a multiplicity of infection of 5 in expansion medium. Cells $\left(1 \times 10^{6}\right.$ cells $/ \mathrm{mL} ; 1 \mathrm{~mL}$ per well in 6-well plates) were transduced three times using spinoculation $(6 \mu \mathrm{g} / \mathrm{mL}$ polybrene, $800 \times g$, $90 \mathrm{~min}$ ) at $6-12 \mathrm{~h}$ intervals for 2 consecutive days. Then cells were washed five times in PBS and transefered in expansion medium containing puromycin $(1 \mu \mathrm{g} / \mathrm{mL})$ for 3 more days.

CFU-C assays. The CFU-C assays were performed by plating 500 or $3000 \mathrm{CD} 34^{+}$ cells per plate in CFU-C media (R\&D Systems, HSC003), according to the manufacturer's instructions. Colonies consisting of at least 40 cells were counted after 15 days at $37^{\circ} \mathrm{C}$ and $5 \% \mathrm{CO}_{2}$. CFU-C colonies were counted blindly regarding control and experimental samples.

Myeloid differentiation. Myeloid lineage-specific $\mathrm{CD} 34^{+}$differentiation was carried out by culturing the cells in expansion media supplemented with $20 \mathrm{ng} / \mathrm{mL}$ GM-CSF (Peprotech, ref. 300-03). Cells were cultured at $1 \times 10^{5}-1 \times 10^{6}$ cells $/ \mathrm{mL}$ densities and analyzed on day 7 and day 14 , using CD34+-PerCP-Cy5.5 (1:60, clone 561), CD11b-PE-Cy7 (1:60, clone ICRF44), CD14-AlexaFluor700 (1:200, clone 63D3), CD16-APC (1:40, clone B73.1), and CD33-PE conjugated antibodies (1:60, clone P67.6) (all from BioLegend). Myeloid differentiation of AML cell lines was assessed using CD11b-PE-Cy7 (1:60, Biolegend clone ICRF44).

Cell viability. Viability was assessed by flow cytometry after staining with Hoechst 33258 (Life Technologies, $1 \mu \mathrm{g} / \mathrm{ml}$; ref. H3569) in PBS supplemented with 2\% FBS and $1 \mathrm{mM}$ EDTA. After $20 \mathrm{~min}$, cells were analyzed in a cell analyzer (BD, LSRFortessa). Viable cells were determined by Hoechst staining exclusion and quantified using FlowJo software (Tree Star, Inc.).

Pharmacological treatments. Zebrafish: For pharmacological treatment, embryos were manually dechorionated and incubated with the PPAR $\delta$ agonist L165,041 (Cayman Chemical, ref. 9000249; $500 \mathrm{nM}$ ) or antagonist GSK3787 (BioVision, ref. $2400 ; 500 \mathrm{nM}$ ) from the 18 somite stage to 48 or $72 \mathrm{hpf}$.

Cells: For mitochondrial membrane potential rescue experiment $\mathrm{CD} 34^{+}$ scrRNA and sh-HLX cells were treated with vehicle (DMSO) or $100 \mathrm{nM}$ PPAR $\delta$ agonist L165,041, while CD34+ GFP and $H L X$ were treated with vehicle (DMSO) or $300 \mathrm{nM}$ PPAR $\delta$ antagonist GSK3787 for $21 \mathrm{~h}$. For the CD34 ${ }^{+}$differentiation experiments, cells were treated with $300 \mathrm{nM}$ antagonist GSK3787 (BioVision, ref. 2897-5), or $5 \mu \mathrm{M}$ Dorsomorphin hydrochloride (Enzo, ref. ENZ-CHM141) for the last $48 \mathrm{~h}$ of the 7-day differentiation protocol, or $10 \mu \mathrm{M}$ Metformin hydrochloride (Sigma, ref. PHR1084) for the last 4 days of the 7-day differentiation. For the HLX knockdown rescue experiments scrRNA and shHLX cells were treated with $100 \mathrm{nM}$ PPAR $\delta$ agonist L165,041 for 7 days. Leukemic cell lines were treated for $48 \mathrm{~h}$ with $1 \mu \mathrm{M}$ PPAR $\delta$ antagonist GSK3787, or $5 \mu \mathrm{M}$ Dorsomorphin.

Detection of autophagic flux. Autophagy was measured using the Cyto-ID Autophagy detection kit (Enzo, ref. ENZ-51031), according to the manufacturer's instructions. Briefly, cells were grown at a density of $1 \times 10^{6}$ cells $/ \mathrm{mL}$ for $16 \mathrm{~h}$ at $37^{\circ} \mathrm{C}$, in the presence of DMSO or Chloroquine $(60 \mu \mathrm{M})$. Cells were washed with PBS, pelleted at $1000 \mathrm{rpm}$ for $5 \mathrm{~min}$, and incubated with cyto-ID green reagent for $30 \mathrm{~min}$ at $37^{\circ} \mathrm{C}$ in the dark prior to flow cytometry analysis on LSRFortessa cell analyzer (BD Biosciences). Mean fluorescence intensity (MFI) was quantified using FlowJo software (Tree Star, Inc.)

Real-time qPCR. Total RNA was extracted from human or zebrafish cells using the RNA Clean \& Concentrator-5 kit (Zymo Reasearch) kit or TRI Reagent, according to the manufacturer's instructions. cDNA was prepared with the SuperScript ${ }^{\mathrm{mm}}$ First-Strand Synthesis System for RT-PCR kit (Thermo Fisher Scientific). qPCR reactions were executed using Fast SYBR green Master Mix (Thermo Fisher Scientific) in a StepOnePlus Real-Time PCR machine (Applied Biosystem). Expression was plotted relative to $R A B 7$ (for human cells) and cyyrl (for zebrafish cells) All qPCR graphs in this study are representative of at least two independent experiments. qPCR primers can be found in Supplementary Table 2.

Metabolic assays. OCR and ECAR were measured using a 96-well XF or XFe extracellular flux analyzer (Seahorse Bioscience) in XF media (non-buffered RPMI medium 1640 containing $25 \mathrm{mM}$ glucose, $2 \mathrm{mM}$ glutamine, and $1 \mathrm{mM}$ sodium pyruvate) containing the cytokine mix of expansion medium for $\mathrm{CD} 34^{+}$experiments or 10\% FBS for zebrafish experiments. The measurements were performed under basal conditions and in response to $1 \mu \mathrm{M}$ oligomycin, $1.5 \mu \mathrm{M}$ FCCP, and $100 \mathrm{nM}$ rotenone combined with $1 \mu \mathrm{M}$ antimycin $\mathrm{A}$.

Glucose tracing. For metabolic tracing THP1 cells were cultured in glucose-free RPMI media with dialyzed serum supplemented with $11 \mathrm{mM} \mathrm{D-[U13C]} \mathrm{glucose}$ and $2 \% \mathrm{P} / \mathrm{S}$ for $24 \mathrm{~h}$. Cells were washed with ice cold $0.9 \% \mathrm{w} / \mathrm{v} \mathrm{NaCl}$ buffer and metabolites were extracted twice with hot ethanol $(70 \%)$ and analyzed by GC mass spectrometry (GCMS). Fractional contribution from exogenous substrates was calculated as described previously ${ }^{69}$. Briefly, metabolite extracts were dried, resuspended in pyridine, and derivatized with methoxylamine and N-tert-Butyldimethylsilyl-N-methyltrifluoroacetamide with $1 \%$ tert-Butyldimethylchlorosilane before GCMS analysis on an Agilent 7890 GC with Agielnt 5977 MS. Peak areas of all possible labeling states were extracted for full-carbon-backbone fragments of selected metabolites to obtain mass distribution vectors (MDVs). MDVs were then corrected for natural abundance of heavy isotopes by constrained optimization of the linear equation

$$
I=L \cdot M
$$

where $I$ denotes the measured fractional abundance of metabolite fragments of different labeling states, $L$ denotes the correction matrix, and $M$ denotes the corrected $\mathrm{MDV}^{69}$. The fractional contribution from exogenous substrates was then calculated as the weighted average of the MDV

$$
\mathrm{FC}=\frac{\sum_{i=0}^{n} i \cdot s_{i}}{n}
$$

where FC denotes the fractional contribution, $i$ is the position in the MDV, $s_{\mathrm{i}}$ is the value of the MDV at the position $i$, and $n$ is the length of the MDV. All calculations were implemented in an in-house $\mathrm{R}$ script.

Mitochondrial membrane potential and mtDNA/nDNA measurement. Zebrafish cells or human cells were stained with tetramethylrhodamine, methyl ester (Image-iT $T^{\mathrm{mox}}$ TMRM Reagent, Invitrogen, ref. I34361; $50 \mathrm{nM}$ ) at 28 or $37^{\circ} \mathrm{C}$, respectively, for $30 \mathrm{~min}$, then washed with PBS-5\% FBS and analyzed by flow cytometry with LSRFortessa or LSR II cell analyzers (BD Biosciences). MFI was quantified using FlowJo software (Tree Star, Inc.). To measure the ratio of mitochondrial and nuclear DNA (mtDNA/nDNA), genomic DNA was extracted from $10^{5}$ cells using DNeasy Blood \& Tissue Kit (Qiagen) and real-time qPCR was performed as described before ${ }^{70}$. Briefly, quantitative PCR reactions were assembled as follows: $2 \mu \mathrm{L}$ of template DNA ( $3 \mathrm{ng} / \mu \mathrm{L}$ isolated DNA), $2 \mu \mathrm{L}$ of mtDNA or nDNA target-specific primer pair ( $400 \mathrm{nM}$ final concentration each), $12.5 \mu \mathrm{L}$ SYBR Green PCR Master Mix, and $8.5 \mu \mathrm{L} \mathrm{H}_{2} \mathrm{O}$ in 1 well of the 96-well PCR plate. All qPCR experiments were performed in triplicate wells in three independent experiments. Primer sequences can be found in Supplementary Table 2. 
Reactive oxygen species. ROS were determined by incubating cells with MitoSOX $^{\mathrm{sw}}$ Red Mitochondrial Superoxide Indicator (Invitrogen, ref. M36008; $5 \mu \mathrm{M}$ ) at $37^{\circ} \mathrm{C}$ for $10 \mathrm{~min}$ in the dark, then washed with PBS-5\% FBS and analyzed by flow cytometry with LSRFortessa or LSR II cell analyzers (BD Biosciences). MFI was quantified using FlowJo software (Tree Star, Inc.).

Western blot. Total cell lysates from all protein samples in this manuscript were incubated on ice in lysis buffer for $20 \mathrm{~min}(50 \mathrm{mM}$ Tris, pH 7.5, $150 \mathrm{mM} \mathrm{NaCl}, 1 \%$ Triton X-100, 10\% glycerol, $1 \mathrm{mM}$ EDTA, $1 \times$ protease and phosphate inhibitors (Sigma-Aldrich)) followed by three cycles of $15 \mathrm{~s}$ sonication (Bioruptor). Lysates were subjected to Western blot analysis to detect Ty tag (1:500, SAB4800032 Sigma-Aldrich), Flag tag (1:5000, Sigma-Aldrich, clone M2, F1804), $\beta$-actin $(1: 10,000, C 4$, Santa Cruz Biotechnology, sc47778), HA tag (1:1000, Sigma-Aldrich, 3F10, 000000011867423001), H3 (1:10.000, Abcam, ab4729), PPAR $\beta$ (1:500, Santa Cruz Biotechnology, F-10, sc-74517), total oxphos (1:2000, Antibody Cocktail, Abcam, ab110413), AMPKa (1:2000, Cell Signaling Technology, D5A2, 5831), and phospho-AMPKa T172 (1:2000, Cell Signaling Technology, 40H9, 2535). For detection of LC3B (1:2000, Cell Signaling Technology, 2775) the cells were additionally subjected to a $24 \mathrm{~h}$ treatment with chloroquine (Sigma-Aldrich, ref. C6628; $25 \mu \mathrm{M}$ ) before lysis. Western blots were either exposed on films or scanned with the BioRad ChemiDoc Touch Imaging System. Uncropped western blots can be found in Supplementary Fig. 8.

Statistical analysis. Statistical analysis was performed using two-tailed unpaired Student's $t$-test or one-way ANOVA test as indicated in the figure legends. Sample sizes and significance are shown in the figure legends. Statistical analysis for the overlap between gene sets was performed with hypergeometric test and shows the significance of the overlap in each case. Other statistical analyses are described in the respective sections.

Genome-wide analysis. RNA-Seq libraries, RNA-Seq preparation and analysis methodology, ATAC-Seq libraries, ATAC-Seq analysis methodology, Chromatin Immunoprecipitation, ChIP-Seq libraries, ChIP-Seq analysis, gene ontology, pathway and network analysis, motif analysis, digital genomic footprinting for ATAC-Seq, network construction, hierarchical differentiation tree can be found in the Supplementary Information.

Code availability. All computer codes used in this manuscript are available upon request.

Data availability. All raw sequencing data have been deposited in the Short Read Archive SRA under the BioProject accession codes PRJNA390228, PRJNA390119, and PRJNA433488. All the data are available without restrictions.

Received: 15 July 2017 Accepted: 29 June 2018

Published online: 06 August 2018

\section{References}

1. Dykstra, B. et al. Long-term propagation of distinct hematopoietic differentiation programs in vivo. Cell Stem Cell 1, 218-229 (2007).

2. Orkin, S. H. \& Zon, L. I. Hematopoiesis: an evolving paradigm for stem cell biology. Cell 132, 631-644 (2008).

3. Osawa, M., Hanada, K., Hamada, H. \& Nakauchi, H. Long-term lymphohematopoietic reconstitution by a single CD34-low/negative hematopoietic stem cell. Science 273, 242-245 (1996).

4. Yamamoto, R. et al. Clonal analysis unveils self-renewing lineage-restricted progenitors generated directly from hematopoietic stem cells. Cell 154, 1112-1126 (2013).

5. Ng, A. P. \& Alexander, W. S. Haematopoietic stem cells: past, present and future. Cell Death Discov. 3, 17002 (2017).

6. Crisan, M. \& Dzierzak, E. The many faces of hematopoietic stem cell heterogeneity. Development 143, 4571-4581 (2016).

7. Eaves, C. J. Hematopoietic stem cells: concepts, definitions, and the new reality. Blood 125, 2605-2613 (2015).

8. Jung, J., Buisman, S. \& de Haan, G. Hematopoiesis during development, aging, and disease. Exp. Hematol. 44, 689-695 (2016).

9. Gottgens, B. Regulatory network control of blood stem cells. Blood 125, 2614-2620 (2015).

10. Daniel, M. G., Pereira, C. F., Lemischka, I. R. \& Moore, K. A. Making a hematopoietic stem cell. Trends Cell Biol. 26, 202-214 (2016).

11. Alharbi, R. A., Pettengell, R., Pandha, H. S. \& Morgan, R. The role of HOX genes in normal hematopoiesis and acute leukemia. Leukemia 27, 1000-1008 (2013).
12. De Braekeleer, E. et al. Hox gene dysregulation in acute myeloid leukemia. Future Oncol. 10, 475-495 (2014).

13. Deguchi, Y. \& Kehrl, J. H. Selective expression of two homeobox genes in CD34-positive cells from human bone marrow. Blood 78, 323-328 (1991)

14. Deguchi, Y., Kirschenbaum, A. \& Kehrl, J. H. A diverged homeobox gene is involved in the proliferation and lineage commitment of human hematopoietic progenitors and highly expressed in acute myelogenous leukemia. Blood 79, 2841-2848 (1992).

15. Hentsch, B. et al. Hlx homeo box gene is essential for an inductive tissue interaction that drives expansion of embryonic liver and gut. Genes Dev. 10, 70-79 (1996).

16. Kehrl, J. H. \& Deguchi, Y. Potential roles for two human homeodomain containing proteins in the proliferation and differentiation of human hematopoietic progenitors. Leuk. Lymphoma 10, 173-176 (1993).

17. Mullen, A. C. et al. Hlx is induced by and genetically interacts with T-bet to promote heritable $\mathrm{T}_{\mathrm{H}} 1$ gene induction. Nat. Immunol. 3, 652-658 (2002).

18. Zhang, Y., Zhang, Y., Gu, W. \& Sun, B. TH1/TH2 cell differentiation and molecular signals. Adv. Exp. Med. Biol. 841, 15-44 (2014).

19. Zheng, W. P. et al. Up-regulation of Hlx in immature Th cells induces IFNgamma expression. J. Immunol. 172, 114-122 (2004).

20. Allen, J. D. et al. Perturbed development of T and B cells in mice expressing an Hlx homeobox transgene. J. Immunol. 154, 1531-1542 (1995).

21. Kawahara, M. et al. H2.0-like homeobox regulates early hematopoiesis and promotes acute myeloid leukemia. Cancer Cell 22, 194-208 (2012).

22. Pandolfi, A. et al. PAK1 is a therapeutic target in acute myeloid leukemia and myelodysplastic syndrome. Blood 126, 1118-1127 (2015).

23. Pandolfi, A. \& Steidl, U. HLX in AML: novel prognostic and therapeutic target. Oncotarget 3, 1059-1060 (2012).

24. Huang, L. et al. Transcription factor Hlx controls a systematic switch from white to brown fat through Prdm16-mediated co-activation. Nat. Commun. 8 , 68 (2017).

25. Herwig, L. et al. Distinct cellular mechanisms of blood vessel fusion in the zebrafish embryo. Curr. Biol. 21, 1942-1948 (2011).

26. Herbert, S. P., Cheung, J. Y. \& Stainier, D. Y. Determination of endothelial stalk versus tip cell potential during angiogenesis by $\mathrm{H} 2.0$-like homeobox-1. Curr. Biol. 22, 1789-1794 (2012).

27. Tamplin, O. J. et al. Hematopoietic stem cell arrival triggers dynamic remodeling of the perivascular niche. Cell 160, 241-252 (2015).

28. Vannini, N. et al. Specification of haematopoietic stem cell fate via modulation of mitochondrial activity. Nat. Commun. 7, 13125 (2016)

29. Ho, T. T. et al. Autophagy maintains the metabolism and function of young and old stem cells. Nature 543, 205-210 (2017).

30. Basak, N. P. \& Banerjee, S. Mitochondrial dependency in progression of acute myeloid leukemia. Mitochondrion 21, 41-48 (2015).

31. Piper, J. et al. Wellington-bootstrap: differential DNase-seq footprinting identifies cell-type determining transcription factors. BMC Genomics 16, 1000 (2015).

32. Ito, K. et al. A PML-PPAR-delta pathway for fatty acid oxidation regulates hematopoietic stem cell maintenance. Nat. Med. 18, 1350-1358 (2012).

33. Fan, W. \& Evans, R. PPARs and ERRs: molecular mediators of mitochondrial metabolism. Curr. Opin. Cell Biol. 33, 49-54 (2015).

34. Wang, P. et al. Peroxisome proliferator-activated receptor \{delta\} is an essential transcriptional regulator for mitochondrial protection and biogenesis in adult heart. Circ. Res. 106, 911-919 (2010).

35. Bagger, F. O. et al. BloodSpot: a database of gene expression profiles and transcriptional programs for healthy and malignant haematopoiesis. Nucleic Acids Res. 44, D917-D924 (2016).

36. Cabezas-Wallscheid, N. et al. Identification of regulatory networks in HSCs and their immediate progeny via integrated proteome, transcriptome, and DNA methylome analysis. Cell Stem Cell 15, 507-522 (2014).

37. Cabezas-Wallscheid, N. et al. Vitamin A-retinoic acid signaling regulates hematopoietic stem cell dormancy. Cell 169, 807-823, (2017).

38. Consortium, E. P. An integrated encyclopedia of DNA elements in the human genome. Nature 489, 57-74 (2012).

39. Corces, M. R. et al. Lineage-specific and single-cell chromatin accessibility charts human hematopoiesis and leukemia evolution. Nat. Genet. 48, 1193-1203 (2016)

40. Zhao, B. et al. Mitochondrial dysfunction activates the AMPK signaling and autophagy to promote cell survival. Genes Dis. 3, 82-87 (2016).

41. Lin, S. C. \& Hardie, D. G. AMPK: sensing glucose as well as cellular energy status. Cell Metab. 27, 299-313 (2017)

42. Herzig, S. \& Shaw, R. J. AMPK: guardian of metabolism and mitochondrial homeostasis. Nat. Rev. Mol. Cell Biol. 19, 121-135 (2018).

43. Hur, K. Y. \& Lee, M. S. New mechanisms of metformin action: focusing on mitochondria and the gut. J. Diabetes Investig. 6, 600-609 (2015).

44. Adhikary, T. et al. The transcriptional PPARbeta/delta network in human macrophages defines a unique agonist-induced activation state. Nucleic Acids Res. 43, 5033-5051 (2015). 
45. Frohling, S. Widespread over-expression of the non-clustered homeobox gene HLX in acute myeloid leukemia. Haematologica 97, 1453 (2012).

46. Liu, T., Chen, J., Xiao, S. \& Lei, X. H2.0-like homeobox 1 acts as a tumor suppressor in hepatocellular carcinoma. Tumour Biol. 37, 6419-6428 (2016).

47. Ito, K. \& Suda, T. Metabolic requirements for the maintenance of selfrenewing stem cells. Nat. Rev. Mol. Cell Biol. 15, 243-256 (2014).

48. Suda, T., Takubo, K. \& Semenza, G. L. Metabolic regulation of hematopoietic stem cells in the hypoxic niche. Cell Stem Cell 9, 298-310 (2011).

49. Mohrin, M. et al. Stem cell aging. A mitochondrial UPR-mediated metabolic checkpoint regulates hematopoietic stem cell aging. Science 347, 1374-1377 (2015).

50. Piccoli, C. et al. To breathe or not to breathe: the haematopoietic stem/ progenitor cells dilemma. Br. J. Pharmacol. 169, 1652-1671 (2013).

51. Takubo, K. et al. Regulation of glycolysis by Pdk functions as a metabolic checkpoint for cell cycle quiescence in hematopoietic stem cells. Cell Stem Cell 12, 49-61 (2013).

52. $\mathrm{Yu}, \mathrm{W}$. M. et al. Metabolic regulation by the mitochondrial phosphatase PTPMT1 is required for hematopoietic stem cell differentiation. Cell Stem Cell 12, 62-74 (2013).

53. Simsek, T. et al. The distinct metabolic profile of hematopoietic stem cells reflects their location in a hypoxic niche. Cell Stem Cell 7, 380-390 (2010).

54. McKenzie, J. L., Takenaka, K., Gan, O. I., Doedens, M. \& Dick, J. E. Low rhodamine 123 retention identifies long-term human hematopoietic stem cells within the Lin-CD34+CD38- population. Blood 109, 543-545 (2007).

55. Piccoli, C. et al. Characterization of mitochondrial and extra-mitochondrial oxygen consuming reactions in human hematopoietic stem cells. Novel evidence of the occurrence of $\mathrm{NAD}(\mathrm{P}) \mathrm{H}$ oxidase activity. J. Biol. Chem. 280, 26467-26476 (2005).

56. Romero-Moya, D. et al. Cord blood-derived CD34+hematopoietic cells with low mitochondrial mass are enriched in hematopoietic repopulating stem cell function. Haematologica 98, 1022-1029 (2013).

57. Guo, B., Huang, X., Lee, M. R., Lee, S. A. \& Broxmeyer, H. E. Antagonism of PPAR-gamma signaling expands human hematopoietic stem and progenitor cells by enhancing glycolysis. Nat. Med. 24, 360-367 (2018).

58. Ito, K. et al. Self-renewal of a purified Tie $2^{+}$hematopoietic stem cell population relies on mitochondrial clearance. Science 354, 1156-1160 (2016).

59. Sriskanthadevan, S. et al. AML cells have low spare reserve capacity in their respiratory chain that renders them susceptible to oxidative metabolic stress. Blood 125, 2120-2130 (2015).

60. Lymboussaki, A. et al. PPARdelta is a ligand-dependent negative regulator of vitamin D3-induced monocyte differentiation. Carcinogenesis 30, 230-237 (2009).

61. Bao, B., Ahmad, A., Azmi, A. S., Ali, S. \& Sarkar, F. H. Overview of cancer stem cells (CSCs) and mechanisms of their regulation: implications for cancer therapy. Curr. Protoc. Pharmacol. 25, doi: 10.1002/0471141755.ph1425s6 (2013).

62. Nakada, D., Saunders, T. L. \& Morrison, S. J. Lkb1 regulates cell cycle and energy metabolism in haematopoietic stem cells. Nature 468, 653-658 (2010).

63. Saito, Y., Chapple, R. H., Lin, A., Kitano, A. \& Nakada, D. AMPK protects leukemia-initiating cells in myeloid leukemias from metabolic stress in the bone marrow. Cell Stem Cell 17, 585-596 (2015)

64. Accordi, B. et al. AMPK inhibition enhances apoptosis in MLL-rearranged pediatric B-acute lymphoblastic leukemia cells. Leukemia 27, 1019-1027 (2013).

65. Sujobert, P. et al. Co-activation of AMPK and mTORC1 induces cytotoxicity in acute myeloid leukemia. Cell Rep. 11, 1446-1457 (2015).

66. Charan, J. \& Kantharia, N. D. How to calculate sample size in animal studies? J. Pharmacol. Pharmacother. 4, 303-306 (2013).

67. Sankaran, V. G. et al. Human fetal hemoglobin expression is regulated by the developmental stage-specific repressor BCL11A. Science 322, 1839-1842 (2008).
68. Moffat, J. et al. A lentiviral RNAi library for human and mouse genes applied to an arrayed viral high-content screen. Cell 124, 1283-1298 (2006).

69. Buescher, J. M. et al. A roadmap for interpreting (13)C metabolite labeling patterns from cells. Curr. Opin. Biotechnol. 34, 189-201 (2015).

70. Rooney, J. P. et al. PCR based determination of mitochondrial DNA copy number in multiple species. Methods Mol. Biol. 1241, 23-38 (2015).

\section{Acknowledgements}

We would like to thank the Deep Sequencing, Imaging, FACS, Fish, and bioinformatics facilities of the MPI for resources and support and David Stachura for valuable protocols. We thank colleagues for reading this manuscript. F.G.K. was supported by a return scholarship of the Forschungskommission, Faculty of Medicine, University of Freiburg, a grant of the Wissenschaftliche Gesellschaft in Freiburg (Scientific Society in Freiburg), and an EXCEL-Fellowship of the Faculty of Medicine, University of Freiburg, funded by the Else-Kröner-Fresenius-Stiftung. E.T. was supported by the Max Planck Society, a Marie Curie Career Integration Grant (631432 Bloody Signals) and the Deutsche Forschungsgemeinschaft, Research Training Group GRK2344 "MeInBio -BioInMe". E.T. and M.W.W. were supported by The Fritz Thyssen Stiftung (Az 10.17.1.026MN).

\section{Author contributions}

I.P. and T.C. performed the bulk of experiments. R.I.K.G., N.Y., X.L., S.L., L.K., P.M., and J.D.C. performed experiments. C.C.A.B. and F.G.K. performed the CHT smear experiments in zebrafish. M.M. made the Tg(UAS:HLX-GFP) and Tg(Mmu.Runx1:GAL4) lines and gave useful advice. A.P. (mainly), D.v.E., and Alex.P. performed the bioinformatics analysis. P.C. performed the digital footprinting analyses. J.M.B. performed metabolomics and analysis. A.R., M.W.W., and E.L.P. provided reagents and critical insights. E. T. conceived that project and wrote the paper together with I.P. and input from all authors.

\section{Additional information}

Supplementary Information accompanies this paper at https://doi.org/10.1038/s41467018-05311-4.

Competing interests: E.L.P. is a founder of Rheos and SAB Member of Immunomet. The remaining authors declare no competing interests.

Reprints and permission information is available online at http://npg.nature.com/ reprintsandpermissions/

Publisher's note: Springer Nature remains neutral with regard to jurisdictional claims in published maps and institutional affiliations.

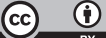

Open Access This article is licensed under a Creative Commons Attribution 4.0 International License, which permits use, sharing, adaptation, distribution and reproduction in any medium or format, as long as you give appropriate credit to the original author(s) and the source, provide a link to the Creative Commons license, and indicate if changes were made. The images or other third party material in this article are included in the article's Creative Commons license, unless indicated otherwise in a credit line to the material. If material is not included in the article's Creative Commons license and your intended use is not permitted by statutory regulation or exceeds the permitted use, you will need to obtain permission directly from the copyright holder. To view a copy of this license, visit http://creativecommons.org/ licenses/by/4.0/

(c) The Author(s) 2018 